\title{
Transverse momentum dependent parton densities in processes with heavy quark generations
}

\author{
A. V. Kotikov, ${ }^{1, *}$ A. V. Lipatov, ${ }^{1,2, \dagger}$ and P. M. Zhang ${ }^{3, *}$ \\ ${ }^{1}$ Joint Institute for Nuclear Research, Dubna 141980, Moscow region, Russia \\ ${ }^{2}$ Skobeltsyn Institute of Nuclear Physics, Lomonosov Moscow State University, Moscow 119991, Russia \\ ${ }^{3}$ School of Physics and Astronomy, Sun Yat-sen University, Zhuhai 519082, China
}

(Received 23 July 2021; accepted 3 September 2021; published 28 September 2021)

\begin{abstract}
We study the heavy quark production processes with the transverse momentum dependent (unintegrated) gluon distribution function in a proton which is obtained recently using the Kimber-Martin-Ryskin prescription from the Bessel-inspired behavior of parton densities at small Bjorken $x$ values. Our results agree with the latest HERA experimental data for reduced cross sections $\sigma_{\text {red }}^{c \bar{c}}\left(x, Q^{2}\right)$ and $\sigma_{\text {red }}^{b \bar{b}}\left(x, Q^{2}\right)$, and also for deep inelastic structure functions $F_{2}^{c}\left(x, Q^{2}\right)$ and $F_{2}^{b}\left(x, Q^{2}\right)$ in a wide range of $x$ and $Q^{2}$ values. Comparisons with the predictions based on the Ciafaloni-Catani-Fiorani-Marchesini evolution equation and with the results of conventional pQCD calculations performed at the first three orders of perturbative expansion are presented.
\end{abstract}

DOI: $10.1103 /$ PhysRevD.104.054042

\section{INTRODUCTION}

Recently, the important new data of the cross sections for the open charm and beauty production in neutral current deep inelastic electron-proton scattering (DIS) have been obtained by combining the results of $\mathrm{H} 1$ and ZEUS Collaborations at HERA [1]. Measurements have shown that heavy flavor production in DIS proceeds predominantly via the photon-gluon fusion process, $\gamma g \rightarrow Q \bar{Q}$, where $Q$ is the heavy quark. The cross section therefore depends strongly on the gluon distribution in the proton and heavy quark mass. Moreover, an analysis of the data in the framework of perturbative quantum chromodynamics (QCD) has been done [1], where the massive fixed-flavornumber scheme and different implementations of the variable-flavor-number scheme were used.

The theoretical description of the heavy quark production processes can also be performed with the transverse momentum dependent (TMD), or unintegrated functions of the density of partons (quarks and/or gluons) in a proton $[2,3]$. These quantities, depending on the fraction $x$ of the longitudinal momentum carried by the parton in the proton, the two-dimensional transverse momentum of the parton $k_{T}^{2}$,

\footnotetext{
*kotikov@theor.jinr.ru

†lipatov@theory.sinp.msu.ru

"zhangpm5@mail.sysu.edu.cn
}

Published by the American Physical Society under the terms of the Creative Commons Attribution 4.0 International license. Further distribution of this work must maintain attribution to the author(s) and the published article's title, journal citation, and DOI. Funded by SCOAP. and the hard scale $\mu^{2}$ of a complex process, contain nonperturbative (including the transverse momentum) information of the proton structure. The TMD factorization theorems provide the necessary basis to separate hard parton physics (which is described in terms of perturbative QCD) and soft parton physics. Currently, there are a number of factorization approaches which include the dependence of the parton distribution function's (PDFs) on the transverse momentum, for example, the Collins-Soper-Sterman [4] approach developed for semi-inclusive processes with a finite and nonzero ratio between the rigid scale $\mu^{2}$ and total energy $s$, as well as the approach to high-energy factorization $[5,6]$ (or $k_{T^{-}}$ factorization [7]) which is valid at a fixed limit of the hard scale and at high energies.

With the high-energy factorization, the TMD density of gluons satisfies the Balitsky-Fadin-Kuraev-Lipatov (BFKL) [8] or Ciafaloni-Catani-Fiorani-Marchesini (CCFM) [9] evolution equations, which resum the contributions of large logarithm terms proportional to $\alpha_{s}^{n} \ln ^{n} s \sim \alpha_{s}^{n} \ln ^{n} 1 / x$. These terms are important at high energy $s$ (or, equivalently, at low $x$ values). Thus, high-order radiative corrections can be effectively taken into account in the cross sections [namely, the part of the next-to-leading order (NLO) + of the nextto-leading order $(\mathrm{NNLO})+\ldots$ terms corresponding to the emission of the original gluons]. Phenomenological applications of the high-energy factorization approach augmented by the CCFM are well known in the literature (see, for example, [10-20] and references therein).

In addition to the CCFM equation, there are also other approaches to determining the TMD PDFs in a proton, namely the parton branching approach (PB) [21] and the Kimber-Martin-Ryskin (KMR) recipe [22] based on the 
usual Dokshitser-Gribov-Lipatov-Altarelli-Parisi (DGLAP) [23] equations. The first one (PB) gives a numerical iterative solution of the DGLAP evolution equations for collinear and TMD PDFs using the concept of resolvable and nonresolvable branching and the Sudakov's formalism to describe the evolution of a parton from one scale to another without decidable branching. The splitting kinematics at each branching vertex is described by the DGLAP equations and the angular ordering condition for parton emission, which can be used instead of the usual DGLAP ordering by virtuality. The KMR approach is a formalism invented to construct TMD PDFs from well-known traditional (collinear) PDFs under the key assumption that the dependence of parton distributions on transverse momentum comes only in the last stage of evolution. It is believed that the KMR procedure effectively takes into account the main part of next-to-leading (NLL) logarithmic terms corresponding to the real gluon emission at the last step of evolution cascade. The KMR approach is currently explored in NLO [24] and commonly used in phenomenological applications (see, for example, [11,13-19] and references therein), where the usual PDFs (for example, the NNPDF [25] or CTEQ [26] ones) are accepted numerically as input. The relationships between $\mathrm{PB}$ and KMR scenarios [27], as well between PB and CCFM approaches [28] have been discussed.

The KMR formalism was used in our recent work [29] for analytical calculations of the TMD PDFs, where we adopted the expressions for usual PDFs obtained with the generalized double asymptotic scaling (DAS) approach [30-33]. The scaling, related to the asymptotic behavior of DGLAP evolution, was discovered many years ago [34]. It was shown [30-32] that the flat initial conditions for the DGLAP equations used in the generalized DAS scheme lead to the Bessel-like behavior of PDFs at small $x$ values. With above results, we obtained the analytical expressions for the TMD quark and gluon densities [29] in the leading order (LO). ${ }^{1}$ In Ref. [29], we have implemented various kinematic constraints that exist in the KMR recipe (namely, angular and strict ordering conditions) and investigated the relationship between the differential and integral formulations of the KMR procedure recently mentioned in [36].

In the present paper we analyze the combined $\mathrm{H} 1$ and ZEUS experimental data [1] for the (reduced) charm and beauty cross sections $\sigma_{\text {red }}^{c \bar{c}}$ and $\sigma_{\text {red }}^{b \bar{b}}$, and charm and beauty contributions to the proton structure functions (SFs) $F_{2}\left(x, Q^{2}\right)$ and $F_{L}\left(x, Q^{2}\right)$ [37-39], as well their ratio for different $Q^{2}$ values. Studying the earlier data on the charm $\mathrm{SF} F_{2}^{c}$ in the proton from H1 [40] and ZEUS [41] Collaborations at HERA for $x \sim 10^{-4}$, it was found that the charm contribution to the total proton $\mathrm{SF} F_{2}$ is about $25 \%$, which is significantly larger than that one found by

\footnotetext{
${ }^{1}$ The obtained TMD PDFs are now implemented in the TMDLIB package [3] and are publicly available. Moreover, they are included in the Monte Carlo event generator PEGASUs [35].
}

the European Muon Collaboration at CERN [42] for large $x$, where SF $F_{2}^{c}$ was only $1 \%$ of $F_{2}$. Such a large value of $F_{2}^{c}$ attracted extensive experimental and theoretical studies of heavy quark production processes (see, for example, the data [1] studied in this paper, as well as the experimental data [43] from LHCb Collaboration at CERN for the prompt charm production in $p p$ collisions). Theoretical studies usually serve to confirm that HERA and LHC data can be described by the perturbative charm generation within (see, for example, reviews [44-46] and references therein). We also note here that, historically, the $k_{T}$-factorization was introduced and tested in the study of these processes (see [5-7]).

The production of charmed mesons at hadron colliders is dominated by the $g g \rightarrow c \bar{c}$ subprocess; therefore it provides a sensitive probe of the gluon density at small $x$. In particular the data [43] of LHCb Collaboration provides the information on the gluon for small $x$ around $x \sim 10^{-6}$ (see [47] and discussions therein). This very small- $x$ region is also crucial for the calculations of signal and background processes for ultra-high energy neutrino astrophysics (see $[48,49]$ for calculations of the high energy neutrino cross section and the prompt atmospheric neutrino flux, respectively). The survey of the heavy quark production will be continued at future lepton-hadron and hadron-hadron colliders, such as LHeC, FCC-eh and FCC-hh, respectively (for a review, see [50,51] and references therein).

To study the process of the heavy quark production, we produce the $k_{T}$-factorization predictions in two ways, namely, the framework of DAS approach and CCFM evolution equation. The direct comparison of these predictions is interesting and could be rather useful to evaluate the TMD parton (mainly gluon) density in a proton. We calculate the high-energy asymptotics of the heavy quark parts of the SFs $F_{2}$ and $F_{L}$ at the first three orders of perturbation theory and present the numerical comparison of these higher-order predictions with corresponding results of the $k_{T}$-factorization calculations.

The outline of our paper is following. In Secs. II and III we briefly describe our theoretical input. Section IV presents our numerical results for the reduced charm and beauty cross sections and charm and beauty parts of SFs $F_{2}\left(x, Q^{2}\right)$ and $F_{L}\left(x, Q^{2}\right)$ in a wide $Q^{2}$ range. Section $\mathrm{V}$ contains our conclusions. In Appendix A we present the high energy asymptotics of the heavy quark contribution to the SFs $F_{2}$ and $F_{L}$ at the first three orders of perturbation theory. Appendix B contains the simple approximations of these formulas for the ratio of the heavy quark parts of the SFs $F_{2}$ and $F_{L}$, which could be useful for subsequent applications.

\section{II. $k_{T}$-DEPENDENT WILSON COEFFICIENT FUNCTIONS}

The differential cross section $\sigma^{Q \bar{Q}}$ (hereafter $Q=c, b$ ) can be presented in the simple form, 
$\frac{d^{2} \sigma^{Q \bar{Q}}}{d x d y}=\frac{2 \pi \alpha^{2}}{x Q^{4}}\left[\left(1-y+\frac{y^{2}}{2}\right) F_{2}^{Q}\left(x, Q^{2}\right)-\frac{y^{2}}{2} F_{L}^{Q}\left(x, Q^{2}\right)\right]$,

where $F_{k}^{Q}\left(x, Q^{2}\right)$ (hereafter $\left.k=2, L\right)$ are heavy quark parts of the proton SFs $F_{k}\left(x, Q^{2}\right), x$ and $y$ are the usual Bjorken scaling variables. Here we present the basic elements of the relations between $\operatorname{SFs}_{2}^{Q}\left(x, Q^{2}\right)$ and $F_{L}^{Q}\left(x, Q^{2}\right)$ and TMD PDFs. More details can be found in [52].

In the $k_{T}$-factorization approach, the SFs $F_{k}^{Q}\left(x, Q^{2}\right)$ are driven at small $x$ by gluons and related in the following way to the TMD gluon distribution $f_{g}\left(x, k_{T}^{2}, \mu^{2}\right)$ :

$$
\begin{aligned}
F_{k}^{Q}\left(x, Q^{2}\right)= & \int_{x}^{1} \frac{d x^{\prime}}{x^{\prime}} \int \frac{d k_{T}^{2}}{k_{T}^{2}} C_{k, g}\left(x, Q^{2}, m^{2}, k_{T}^{2}, \mu^{2}\right) \\
& \times f_{g}\left(\frac{x}{x^{\prime}}, k_{T}^{2}, \mu^{2}\right) .
\end{aligned}
$$

The functions $C_{k, g}\left(x, Q^{2}, m_{f}^{2}, k_{T}^{2}, \mu^{2}\right)$ are regarded as the structure functions of the off shell gluons with virtuality $k_{T}^{2}$ (hereafter we call them as Wilson coefficient functions). Following [52], we do not use the Sudakov decomposition, which is sometimes quite convenient in high-energy calculations. Here we only note that the property between four-dimensional $k^{2}$ and $k_{T}^{2}$, i.e., $k^{2}=-k_{T}^{2}$, comes from the fact that the Bjorken parton variable $x$ in the standard and in the Sudakov approaches coincide.

The $k_{T}$-dependent Wilson coefficient functions have the following form:

$$
C_{k, g}(x)=\frac{1}{\tilde{\beta}^{4}}\left[\tilde{\beta}^{2} C_{k, g}^{F}(x)-12 b x^{2} \frac{q^{\alpha} q^{\beta}}{Q^{2}} C_{k, g}^{A}(x)\right],
$$

where $C_{k, g}^{F}(x)$ and $C_{k, g}^{A}(x)$ corresponds to the application of the Feynman $P_{F}^{\alpha \beta}$ polarization tensor and additional tensor of the gluon polarization $P_{A}^{\alpha \beta}$ (see Ref. [52]),

$$
\hat{P}_{F}^{\alpha \beta}=-\frac{1}{2} g^{\alpha \beta}, \quad \hat{P}_{A}^{\alpha \beta}=\frac{6 b x^{2}}{\tilde{\beta}^{2}} \frac{q^{\alpha} q^{\beta}}{Q^{2}} .
$$

Hereafter,

$\tilde{\beta}^{2}=1-4 b x^{2}, \quad b=-\frac{k^{2}}{Q^{2}} \equiv \frac{k_{T}^{2}}{Q^{2}}, \quad a=\frac{m^{2}}{Q^{2}}, \quad Q^{2}>0$,

and we omitted the dependence of the coefficient functions on the heavy quark mass $m, Q^{2}, k_{T}^{2}$ and hard scale $\mu^{2}$.

The results for the coefficient functions $C_{k, g}(x)$ have been calculated in Ref. [52], which have the following form $(j=F, A)$ :

$$
\begin{gathered}
C_{2, g}^{j}(x)=\frac{e_{Q}^{2} a_{s}}{\tilde{\beta}^{2}}\left[f_{j}^{(1)}+\frac{3}{2 \tilde{\beta}^{2}} f_{j}^{(2)}\right] \Theta\left(x_{1}-x\right) \\
C_{L, g}^{j}(x)=\frac{e_{Q}^{2} a_{s}}{\tilde{\beta}^{2}}\left[4 b x^{2} f_{j}^{(1)}+\frac{\left(1+2 b x^{2}\right)}{\tilde{\beta}^{2}} f_{j}^{(2)}\right] \Theta\left(x_{1}-x\right) \\
=\frac{e_{Q}^{2} a_{s}}{\tilde{\beta}^{2}} f_{j}^{(2)} \Theta\left(x_{1}-x\right)+4 b x^{2} C_{2, g}^{j}
\end{gathered}
$$

where $a_{s}=\alpha_{s} /(4 \pi)$ is the strong coupling constant, and $\Theta\left(x_{1}-x\right)$ is the Heaviside step function with

$$
x_{1}=\frac{1}{1+4 a+b} .
$$

The functions $f_{j}^{(i)}(i=1,2)(J=A, F)$ in the r.h.s. of Eqs. (6) and (7) have the following form

$$
\begin{aligned}
f_{F}^{(1)}= & -2 \bar{\beta}\left[1-(1-2 x(1+b-2 a)[1-x(1+b+2 a)]) f_{1}\right. \\
& \left.+(2 a-b)(1-2 a) x^{2} f_{2}\right], \\
f_{F}^{(2)}= & 8 x \bar{\beta}[(1-(1+b) x) \\
& -2 x\left(b x(1-(1+b) x)(1+b-2 a)+a \tilde{\beta}^{2}\right) f_{1} \\
& \left.+b x^{2}(1-(1+b) x)(2 a-b) f_{2}\right] \\
f_{A}^{(1)}= & -\bar{\beta}\left[\frac{1-x(1+b)}{x}\right. \\
& -2\left(x(1-x(1+b))(1+b-2 a)+a \tilde{\beta}^{2}\right) f_{1} \\
& \left.-x(1-x(1+b))(1-2 a) f_{2}\right],
\end{aligned}
$$

$f_{A}^{(2)}=4 \bar{\beta}(1-(1+b) x)^{2}\left[2-\left(1+2 b x^{2}\right) f_{1}-b x^{2} f_{2}\right]$

and

$\bar{\beta}^{2}=1-\frac{4 a x}{(1-(1+b) x)}, \quad f_{1}=\frac{1}{\tilde{\beta} \bar{\beta}} \ln \frac{1+\bar{\beta} \tilde{\beta}}{1-\bar{\beta} \tilde{\beta}}$,

$f_{2}=\frac{-4}{1-\bar{\beta}^{2} \tilde{\beta}^{2}}$.

\section{A. The case of on shell gluons}

In the particular case of on shell initial gluons, when $k_{T}^{2}=0$, we have (see [52] for more details),

$$
C_{k, g}(x)=e_{Q}^{2} a_{s}\left(\mu^{2}\right) B_{k, g}^{(0)}(x, a),
$$

where 


$$
\begin{aligned}
B_{2, g}^{(0)}(x, a)= & x\left[f^{(1)}(x, a)+\frac{3}{2} f^{(2)}(x, a)\right] \\
= & -2 x \beta[(1-4 x(2-a)(1-x)) \\
& -\left(1-2 x(1-2 a)+2 x^{2}\left(1-6 a-4 a^{2}\right) L(\beta)\right],
\end{aligned}
$$

$$
B_{L, g}^{(0)}(x, a)=x f^{(2)}(x, a)=8 x^{2} \beta[(1-x)-2 x a L(\beta)]
$$

with $f^{(i)}(x, a)=f_{F}^{(i)}(x, a, b=0)(i=1,2)$ and

$$
\beta^{2}=\bar{\beta}^{2}(b=0)=1-\frac{4 a x}{(1-x)}, \quad L(\beta)=\frac{1}{\beta} \ln \frac{1+\beta}{1-\beta} .
$$

Here $B_{k, g}^{(0)}(x, a)$ is the LO collinear Wilson coefficient function. Equations (14) and (15) coincide with the results [53].

\section{KIMBER-MARTIN-RYSKIN APPROACH}

Here we present the main elements of TMD PDFs, based on the KMR prescription in so-called integral formulation (see [36]) and the DAS approach for usual PDFs. More details, including the differential formulation of the KMR prescription, can be found in our previous paper [29].

The TMD quark and gluon distributions (hereafter $a=q, g)$,

$$
\begin{aligned}
f_{a}\left(x, k_{T}^{2}, \mu^{2}\right)= & T_{a}\left(\mu^{2}, k_{T}^{2}\right) \sum_{a^{\prime}} \int_{x}^{x_{0}} \frac{d z}{z} P_{a a^{\prime}}\left(z, k_{T}^{2}\right) \\
& \times D_{a}\left(\frac{x}{z}, k_{T}^{2}\right), \quad x_{0}=1-\Delta,
\end{aligned}
$$

where $D_{a}\left(x, \mu^{2}\right)$ are the conventional PDFs, $f_{a}\left(x, \mu^{2}\right)=$ $x D_{a}\left(x, \mu^{2}\right), T_{a}\left(\mu^{2}, k_{T}^{2}\right)$ are the Sudakov form factors and $P_{a a^{\prime}}\left(z, \mu^{2}\right)$ are the DGLAP splitting functions (see, for example, (2.56)-(2.60) in [54]),

$$
P_{a a^{\prime}}\left(z, \mu^{2}\right)=2 a_{s}\left(\mu^{2}\right) P_{a a^{\prime}}^{(\mathrm{LO})}(z)+\cdots
$$

\section{A. Sudakov form factors $T_{a}\left(\mu^{2}, k_{T}^{2}\right)$}

The Sudakov form factor $T_{a}\left(\mu^{2}, k_{T}^{2}\right)$ has the following form [see (2.4) in[36]]:

$$
T_{a}\left(\mu^{2}, k_{T}^{2}\right)=\exp \left\{-\int_{k_{T}^{2}}^{\mu^{2}} \frac{d p^{2}}{p^{2}} \sum_{a^{\prime}} \int_{0}^{x_{0}} d z z P_{a^{\prime} a}\left(z, p^{2}\right)\right\}
$$

When $\Delta$ is a constant, we have

$$
T_{a}\left(\mu^{2}, k_{T}^{2}\right)=\exp \left[-d_{a} R_{a}(\Delta) s_{1}\right]
$$

where

$$
\begin{aligned}
s_{1} & =\ln \left(\frac{a_{s}\left(k_{T}^{2}\right)}{a_{s}\left(\mu^{2}\right)}\right), \quad d_{a}=\frac{4 C_{a}}{\beta_{0}}, \quad C_{q}=C_{F}, \quad C_{g}=C_{A}, \quad \beta_{0}=\frac{C_{A}}{3}(11-2 \varphi), \quad \varphi=\frac{f}{C_{A}}=\frac{f}{3}, \\
R_{q}(\Delta) & =\ln \left(\frac{1}{\Delta}\right)-\frac{3 x_{0}^{2}}{4},
\end{aligned}
$$

and $C_{A}=N_{c}, C_{F}=\left(N_{c}^{2}-1\right) /\left(2 N_{c}\right)$ for the color $S U\left(N_{c}\right)$ group and $f$ is the number of active quarks.

\section{B. Conventional PDFs}

At LO, the conventional sea quark and gluon densities $f_{a}\left(x, \mu^{2}\right)$ can be written as follows:

$$
\begin{aligned}
& f_{a}\left(x, \mu^{2}\right)=f_{a}^{+}\left(x, \mu^{2}\right)+f_{a}^{-}\left(x, \mu^{2}\right), \\
& f_{g}^{+}\left(x, \mu^{2}\right)=\left(A_{g}+C A_{q}\right) I_{0}(\sigma) e^{-\bar{d}_{+} s}+O(\rho), \quad C=\frac{C_{F}}{C_{A}}=\frac{4}{9}, \\
& f_{q}^{+}\left(x, \mu^{2}\right)=\frac{\varphi}{3}\left(A_{g}+C A_{q}\right) \rho I_{1}(\sigma) e^{-\bar{d}_{+} s}+O(\rho), \\
& f_{g}^{-}\left(x, \mu^{2}\right)=-C A_{q} e^{-d_{-} s}+O(x), \quad f_{q}^{-}\left(x, \mu^{2}\right)=A_{q} e^{-d_{-} s}+O(x),
\end{aligned}
$$

where $I_{\nu}(\sigma)(\nu=0,1)$ are the modified Bessel functions. And

$$
s=\ln \left(\frac{a_{s}\left(Q_{0}^{2}\right)}{a_{s}\left(\mu^{2}\right)}\right), \quad a_{s}\left(\mu^{2}\right)=\frac{1}{\beta_{0} \ln \left(\mu^{2} / \Lambda_{\mathrm{LO}}^{2}\right)}, \quad \sigma=2 \sqrt{\left|\hat{d}_{+}\right| s \ln \left(\frac{1}{x}\right)}, \quad \rho=\frac{\sigma}{2 \ln (1 / x)}
$$


and

$$
\hat{d}_{+}=-\frac{4 C_{A}}{\beta_{0}}, \quad \bar{d}_{+}=1+\frac{4 f(1-C)}{3 \beta_{0}}, \quad d_{-}=\frac{4 C f}{3 \beta_{0}}
$$

are the singular and regular parts of the anomalous dimensions, $\beta_{0}$ is the first coefficient of the QCD $\beta$-function in the $\overline{\mathrm{MS}}$-scheme. The results for the parameters $A_{a}$ and $Q_{0}^{2}$ can be found in $[30,55]$ with $\alpha_{s}\left(M_{Z}\right)=0.1168$.

\section{TMDs in KMR approach}

Now we can use (17) to find the results for TMDs without derivatives. After some algebra we have

$$
\begin{aligned}
f_{a}\left(x, k_{T}^{2}, \mu^{2}\right)= & 4 C_{a} a_{s}\left(k_{T}^{2}\right) T_{a}\left(\mu^{2}, k_{T}^{2}\right) \\
& \times\left(D_{a}(\Delta) f_{a}\left(\frac{x}{x_{0}}, k_{T}^{2}\right)+D_{a}^{+} f_{a}^{+}\left(\frac{x}{x_{0}}, k_{T}^{2}\right)+D_{a}^{-} f_{a}^{-}\left(\frac{x}{x_{0}}, k_{T}^{2}\right)\right) .
\end{aligned}
$$

Also we can obtain (see more details in [29])

$$
\begin{aligned}
D_{q}(\Delta)=\ln \left(\frac{1}{\Delta}\right)-\frac{x_{0}}{4}\left(2+x_{0}\right), & D_{g}(\Delta)=\ln \left(\frac{1}{\Delta}\right)-x_{0}+\frac{x_{0}^{2}}{4}-\frac{x_{0}^{3}}{3}, \\
D_{q}^{-}(\Delta) & =-\frac{x_{0} \varphi}{2}\left(1-x_{0}+\frac{2 x_{0}^{2}}{3}\right), \quad D_{g}^{-}(\Delta)=0, D_{g}^{+}=\frac{1}{\bar{\rho}_{g}}-x_{0}+\frac{x_{0}^{2}}{4}+\frac{C \varphi}{3}, \\
D_{q}^{+} & =\frac{3 x_{0}}{2 C}\left[\frac{1}{\bar{\rho}_{s}}\left(1-x_{0}+\frac{2 x_{0}^{2}}{3}\right)-\left(1-\frac{x_{0}}{2}+\frac{2 x_{0}^{2}}{9}\right)\right],
\end{aligned}
$$

where

$$
\frac{1}{\rho_{g}}=\frac{1}{\rho} \frac{I_{1}(\sigma)}{I_{0}(\sigma)}, \quad \frac{1}{\rho_{q}}=\frac{1}{\rho} \frac{I_{0}(\sigma)}{I_{1}(\sigma)}, \quad \bar{\sigma}=\sigma\left(x \rightarrow \frac{x}{x_{0}}\right), \quad \bar{\rho}_{a}=\rho_{a}\left(x \rightarrow \frac{x}{x_{0}}\right) .
$$

\section{Other prescriptions}

1. For the phenomenological applications, we use the cutoff parameter $\Delta$ in the angular ordering [36] (the case of strong ordering can be found in [29]),

$$
\Delta_{\text {ang }}=\frac{k_{T}}{k_{T}+\mu} .
$$

In all above cases, except the results for $T_{a}\left(\mu^{2}, k_{T}^{2}\right)$, we can simply replace the parameter $\Delta$ by $\Delta_{\text {ang. }}$. For the Sudakov form factors, we note that the parameters $\Delta$ contribute to the integrand in (19); therefore the momentum dependence changes the results in (20). To perform the correct evaluation of the integral (19), we should recalculate the $p^{2}$ integration in (19),

$T_{a}^{(\text {ang })}\left(\mu^{2}, k_{T}^{2}\right)=\exp \left[-4 C_{a} \int_{k_{T}^{2}}^{\mu^{2}} \frac{d p^{2}}{p^{2}} a_{s}\left(p^{2}\right) R_{a}\left(\Delta_{\text {ang }}\right)\right]$.

The analytic evaluation of $T_{a}^{(\text {ang })}\left(\mu^{2}, k_{T}^{2}\right)$ is a very cumbersome procedure, which will be accomplished in the future. In the following we use the numerical results for $T_{a}^{(\text {ang })}\left(\mu^{2}, k_{T}^{2}\right)$.

2. As it was shown [32], the fits of the experimental data for SF $F_{2}$ are not very well at low $Q^{2}$ values. To solve this problem, one can modify the strong-coupling constant in the infrared region [30]. Specifically, usually there are two kinds of modifications: the "frozen" coupling constant (see, for example, $[30,56]$ ) and the analytic one [57,58], which effectively increase the strong coupling constant argument at small $\mu^{2}$ values, in accordance with [59,60]. As one can see from [30,55,61], both modifications can describe the $F_{2}\left(x, Q^{2}\right)$ data in the small $Q^{2}$ range better than the canonical fit significantly. However, the "frozen" coupling constant leads to a better agreements with data sets as shown in [29]. Thus, we will use it in our present analysis. Let us introduce the freezing of the strong coupling constant by changing it as $\mu^{2} \rightarrow \mu^{2}+M_{\rho}^{2}$, where $M_{\rho}$ is the $\rho$ meson mass [56]. Then, in the formulas of Sec. III we introduce the following replacement:

$$
\alpha_{s}\left(\mu^{2}\right) \rightarrow \alpha_{\mathrm{fr}}\left(\mu^{2}\right)=\alpha_{s}\left(\mu^{2}+M_{\rho}^{2}\right) .
$$


3. In the phenomenological applications (see Sec. IV), the calculated TMD parton densities will be used to predict the reduced cross sections $\sigma_{\text {red }}^{Q \bar{Q}}$ and the proton SFs $F_{k}^{Q}\left(x, Q^{2}\right)$. According to the $k_{T}$-factorization theorem $[5,7]$, the theoretical predictions for these observables can be obtained by convolution (2) of the TMD gluon densities and corresponding off shell production amplitudes. So, we need the TMD quark and gluon distributions in rather broad range of the $x$ variable, i.e., beyond the standard $x$ range $(x \leq 0.05)$.

It was shown (see [29] and discussions therein) that the analytic expressions for TMD parton densities can be modified in the form,

$$
f_{a}\left(x, k_{T}^{2}, \mu^{2}\right) \rightarrow f_{a}\left(x, k_{T}^{2}, \mu^{2}\right)\left(1-\frac{x}{x_{0}}\right)^{\beta_{a}(s)},
$$

which is in agreement with a similar modifications of conventional PDFs (see, for example, the recent paper [62], where the similar extension has been done in the case of the EMC effect from the study of shadowing [63] at low $x$ to antishadowing effect at $x \sim 0.1-0.2)$. The value of $\beta_{a}(0)$ can be estimated from the quark counting rules [64],

$$
\begin{aligned}
& \beta_{v}(0) \sim 3, \quad \beta_{g}(0) \sim \beta_{v}(0)+1 \sim 4, \\
& \beta_{q}(0) \sim \beta_{v}(0)+2 \sim 5,
\end{aligned}
$$

where $v$ marks the valence part of quark density. Usually $\beta_{v}(0), \beta_{g}(0), \beta_{q}(0)$ are determined from fits of experimental data (see, for example [65-67]). In our analysis, we use the numerical values of $\beta_{g}(0)=3.03$ which have been extracted [29] from the fit to the inclusive $b$-jet production data taken by the CMS [68] and ATLAS [69] Collaborations in $p p$ collisions at $\sqrt{s}=7 \mathrm{TeV}$.

\section{PHENOMENOLOGICAL APPLICATIONS}

We are now in a position to apply the TMD parton densities, obtained in [29] and shown above, for phenomenological applications. In the present paper we consider the reduced charm and beauty cross sections $\sigma_{\text {red }}^{c \bar{c}}$ and $\sigma_{\text {red }}^{b \bar{b}}$ and charm and beauty contributions to the deep inelastic proton $\mathrm{SFs} F_{2}\left(x, Q^{2}\right)$, which are directly related with the gluon content of the proton. The observables were measured in $e p$ collisions at HERA with a rather good accuracy (see [1] and [37-39]). In the following we will use latest TMD gluon density in a proton, obtained from the numerical solution of the CCFM evolution equation, namely, JH'2013 set 2 one [70]. Our choice is motivated mainly by the fact that the CCFM equation smoothly interpolates between the small- $x$ BFKL gluon dynamics and conventional DGLAP one, as it was mentioned above. The input parameters of starting (initial) gluon distribution implemented into the JH'2013 set 2 were fitted to describe the high-precision DIS data on structure functions
$F_{2}\left(x, Q^{2}\right)$ and $F_{2}^{c}\left(x, Q^{2}\right)$ at $x \leq 5 \times 10^{-3}$ (see [70] for more information). Of course, we use the same coefficient functions (6) and (7) when we calculate the charm and beauty reduced cross sections and proton structure functions with the CCFM-evolved TMD gluon density. Everywhere below, we always fix the charm and beauty masses to $m_{c}=1.65 \mathrm{GeV}$ and $m_{b}=4.78 \mathrm{GeV}$ [71]. We use the one-loop formula for the QCD coupling $\alpha_{s}$ with $n_{f}=4$ quark flavors at $\Lambda_{\mathrm{QCD}}=143 \mathrm{MeV}$ [that corresponds to $\alpha_{s}\left(m_{Z}^{2}\right)=0.1168$ ] for the analytically calculated TMD gluon density as described above. In the case of CCFM-evolved gluon, we apply the two-loop expression for $\alpha_{s}$ with $n_{f}=4$ and $\Lambda_{\mathrm{QCD}}=200 \mathrm{MeV}$, as it is fixed by the fit [70].

\section{A. Reduced cross sections $\sigma_{\text {red }}^{Q \bar{Q}}$ and SFs $F_{k}^{Q}\left(x, Q^{2}\right)$}

Usually the differential cross section (1) of heavy quark production in the deep inelastic scattering is represented in terms of reduced cross sections $\sigma_{\text {red }}^{Q \bar{Q}}$, which are defined as follows:

$$
\frac{d^{2} \sigma^{Q \bar{Q}}}{d x d y}=\frac{2 \pi \alpha^{2}}{x Q^{4}}\left(1-y+\frac{y^{2}}{2}\right) \sigma_{\text {red }}^{Q \bar{Q}}
$$

Hence, with (1) and (33) the reduced cross section $\sigma_{\text {red }}^{Q \bar{Q}}$ can be easily rewritten through $F_{2}^{Q}\left(x, Q^{2}\right)$ and $F_{L}^{Q}\left(x, Q^{2}\right)$ as

$$
\begin{aligned}
\sigma_{\text {red }}^{Q \bar{Q}} & =F_{2}^{Q}\left(x, Q^{2}\right)-\frac{y^{2}}{1+(1-y)^{2}} F_{L}^{Q}\left(x, Q^{2}\right) \\
& =F_{2}^{Q}\left(x, Q^{2}\right)\left(1-\frac{y^{2}}{1+(1-y)^{2}} \bar{R}^{Q}\left(x, Q^{2}\right)\right),
\end{aligned}
$$

where the ratio $\bar{R}^{Q}\left(x, Q^{2}\right)$ in defined as

$$
\bar{R}^{Q}\left(x, Q^{2}\right)=\frac{F_{L}^{Q}\left(x, Q^{2}\right)}{F_{2}^{Q}\left(x, Q^{2}\right)} .
$$

The evaluation below is based on the formulas (34) and (2) with the coefficient functions as given by (6)-(12).

Our numerical results for reduced cross sections $\sigma_{\text {red }}^{c \bar{c}}$ and $\sigma_{\text {red }}^{b \bar{b}}$ are shown in Figs. 1 and 2, respectively, in comparison with the latest $\mathrm{H} 1$ and ZEUS data [1]. The shaded bands represent the theoretical uncertainties of our calculations. We find that the $k_{T}$-factorization predictions obtained using derived analytical expressions for TMD gluon density in a proton are in perfect agreement with the HERA data in a wide region of $x$ and $Q^{2}$ within the theoretical and experimental uncertainties, both in normalization and shape. These results tend to slightly overshoot the JH'2013 set 2 predictions in the region of small $x$ and especially at low $Q^{2}$. At larger $Q^{2}$ and/or moderate or large $x \geq 10^{-2}$ the CCFM-evolved gluon density tends to 


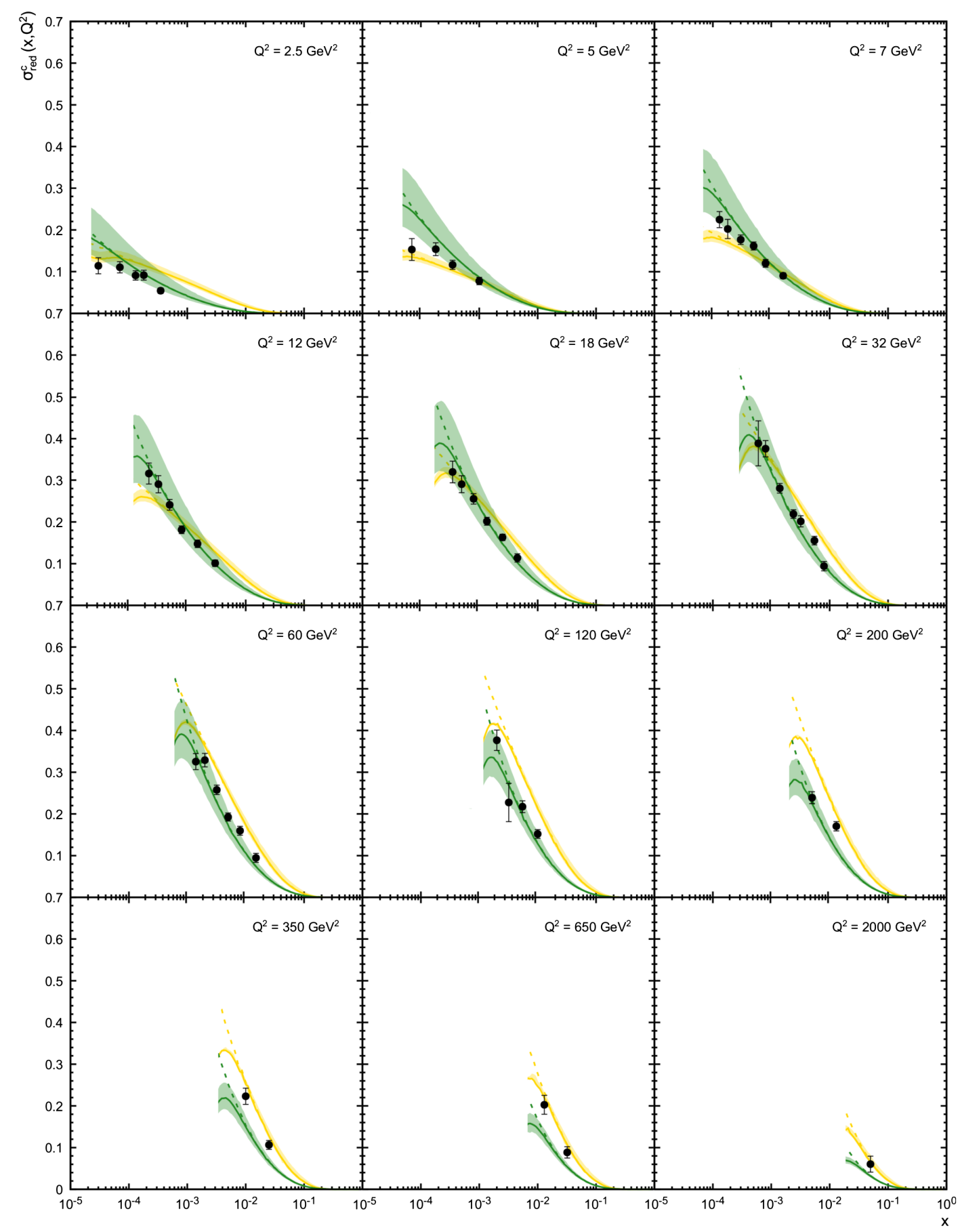

FIG. 1. The reduced charm cross sections $\sigma_{\text {red }}^{c \bar{c}}\left(x, Q^{2}\right)$ as a function of $x$ calculated at different $Q^{2}$ values. The predictions obtained with analytical TMD gluon density in a proton and CCFM-evolved one are shown by the solid green and yellow curves, respectively. The shaded bands correspond to the scale uncertainties of our calculations. The dashed curves represent the contributions from SF $F_{2}^{c}\left(x, Q^{2}\right)$, as it is described in the text. The experimental data are from H1 and ZEUS [1].

overestimate the HERA data, which could be understood by the determination of corresponding input parameters at small $x$ only (see [70]). To estimate the scale uncertainties we introduce the standard variations (by a factor of 2) in default renormalization and factorization scales, which are set to be equal to $\mu_{R}^{2}=4 m_{Q}^{2}+Q^{2}$ and $\mu_{F}^{2}=Q^{2}$, respectively. To show the contribution of the longitudinal structure functions
$F_{L}^{c}\left(x, Q^{2}\right)$ and $F_{L}^{b}\left(x, Q^{2}\right)$, we present also the results for $F_{2}^{c}\left(x, Q^{2}\right)$ and $F_{2}^{b}\left(x, Q^{2}\right)$ as dotted curves in Figs. 1 and 2. The difference between the estimated $\sigma_{\text {red }}^{Q \bar{Q}}$ and $F_{2}^{Q}\left(x, Q^{2}\right)$ is due to the contribution of the longitudinal SFs $F_{L}^{Q}\left(x, Q^{2}\right)$, as it can been clearly seen from (34). So, our calculations show that these contributions are rather important at low $x$. 


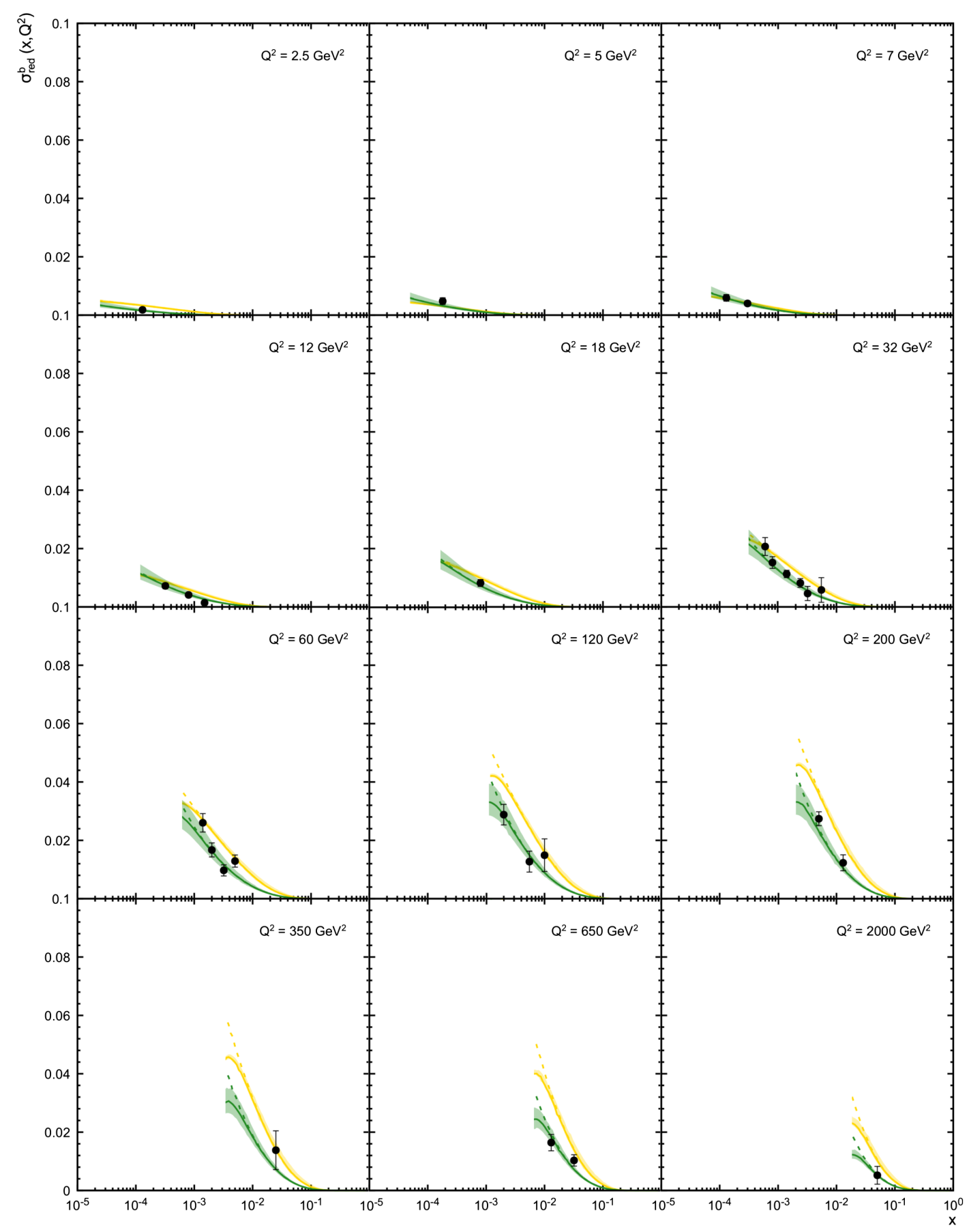

FIG. 2. The reduced beauty cross sections $\sigma_{\text {red }}^{b \bar{b}}\left(x, Q^{2}\right)$ as a function of $x$ calculated at different $Q^{2}$ values. Notation of all curves is the same as in Fig. 1. The experimental data are from H1 and ZEUS [1].

In order to show the difference between $\sigma_{\text {red }}^{Q \bar{Q}}$ and $F_{2}^{Q}\left(x, Q^{2}\right)$ more clearly, in Figs. 3 and 4 we compare our results for SFs $F_{2}^{c}\left(x, Q^{2}\right)$ and $F_{2}^{b}\left(x, Q^{2}\right)$ with the latest ZEUS [37] and H1 [38,39] data. Our predictions for the reduced cross sections $\sigma_{\text {red }}^{c \bar{c}}$ and $\sigma_{\text {red }}^{b \bar{b}}$ are presented here as dotted curves. One can see again that the results obtained with analytically evaluated TMD gluon density are in good agreement with the latest HERA data for both structure functions $F_{2}^{c}\left(x, Q^{2}\right)$ and $F_{2}^{b}\left(x, Q^{2}\right)$ in a wide region of $x$ and $Q^{2}$. The CCFM-evolved gluon JH'2013 set 2 provides a bit worse description of the HERA data, although these results are rather close to the measurements. We find that the discrepancy between two considered approaches tends to be more clearly pronounced at large $Q^{2}$. 


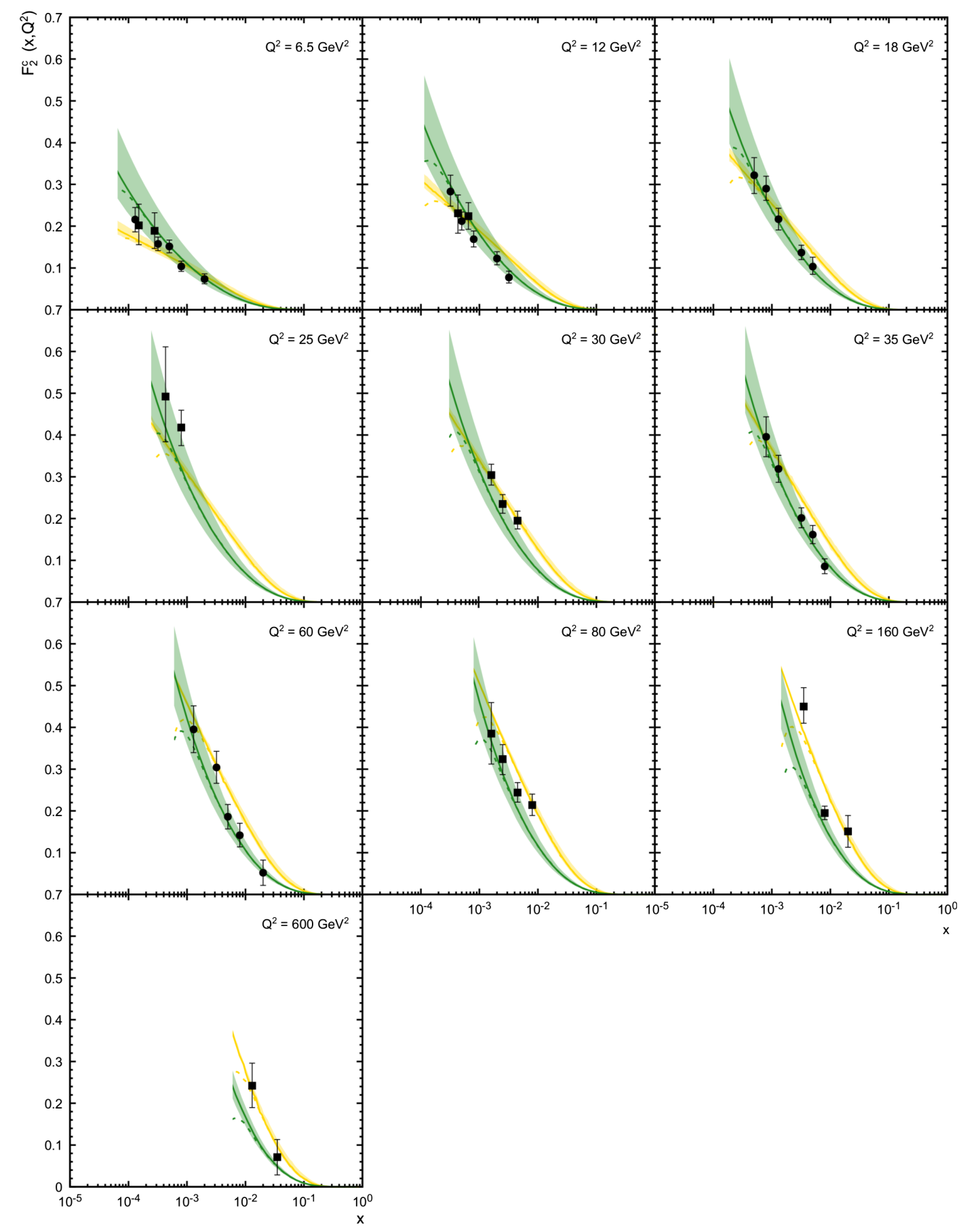

FIG. 3. The charm contribution to the proton structure function $F_{2}\left(x, Q^{2}\right)$ as a function of $x$ calculated at different $Q^{2}$ values. Notation of all curves is the same as in Fig. 1, except that the solid and dotted lines represent contributions from $F_{2}^{c}\left(x, Q^{2}\right)$ and $\sigma_{\text {red }}^{c \bar{c}}\left(x, Q^{2}\right)$ respectively. The experimental data are from ZEUS [37] and H1 [38].

\section{B. Ratio $\bar{R}^{Q}\left(x, Q^{2}\right)$}

Following the results of [52] and using our coefficient functions obtained in Sec. II and TMD gluon density presented in Sec. III, now we can produce predictions for the ratio $\bar{R}^{Q}\left(x, Q^{2}\right)$ according to (35). Results for $\bar{R}^{c}\left(x, Q^{2}\right)$ are presented in Fig. 5, where we plot this ratio as a function of $x$ in a wide $Q^{2}$ range. As earlier, we have applied two TMD gluon densities in a proton discussed above. $^{2}$

Our calculations indicate approximately flat (independent of $x$ ) behavior of $\bar{R}^{c}\left(x, Q^{2}\right)$ with $\bar{R}^{c} \sim 0.1$ at low

\footnotetext{
${ }^{2}$ The predictions for the ratio $\bar{R}^{b}\left(x, Q^{2}\right)$ are rather similar and not shown here.
} 


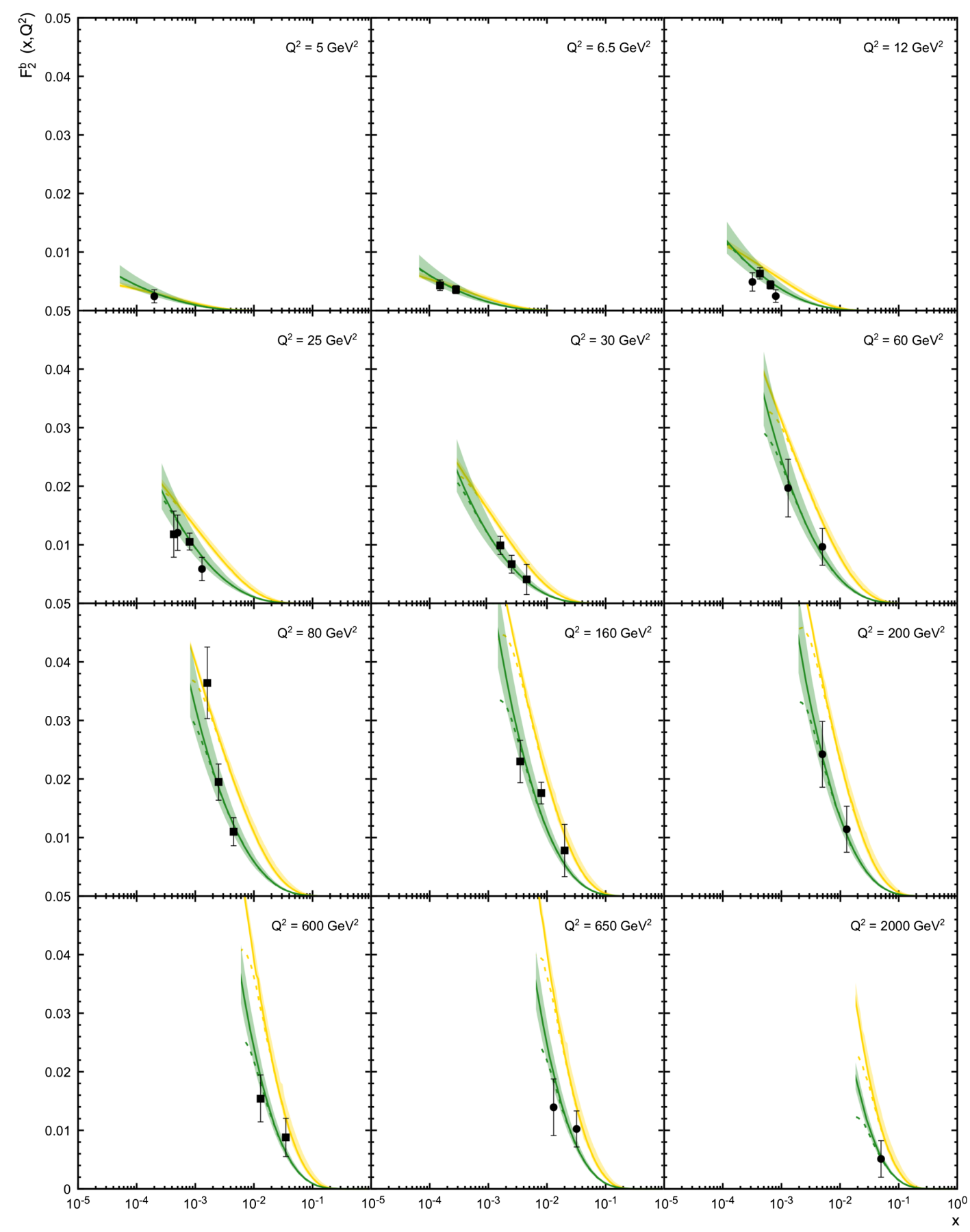

FIG. 4. The beauty contribution to the proton structure function $F_{2}\left(x, Q^{2}\right)$ as a function of $x$ calculated at different $Q^{2}$ values. Notation of all curves is the same as in Fig. 2, except that the solid and dotted lines represent contributions from $F_{2}^{b}\left(x, Q^{2}\right)$ and $\sigma_{\text {red }}^{b \bar{b}}\left(x, Q^{2}\right)$ respectively. The experimental data are from ZEUS [37] and H1 [39].

$Q^{2} \sim 5 \mathrm{GeV}^{2}$ and $\bar{R}^{c} \sim 0.3-0.4$ at higher $Q^{2} \sim 200 \mathrm{GeV}^{2}$. The results obtained with our TMD gluon and CCFMevolved one are in a good agreement to each other. The difference between them is visible at very large $Q^{2}$ only. Moreover, the obtained predictions are in good agreement with [52], which were obtained with the rather old representations for the TMD gluon density (see [72] and more recent [3]).
Next, we would like to compare the results for the ratio $\bar{R}^{c}\left(x, Q^{2}\right)$, obtained in $k_{T}$-factorization with the one $\hat{R}^{c}\left(x, Q^{2}\right)$ [see (A4)], where the ratio $\hat{R}^{c}\left(x, Q^{2}\right)$ was obtained in the conventional (collinear) QCD factorization at first three orders of perturbation theory (see Appendix A) represented by the solid, dashed and dotted gray curves in Fig. 5. To evaluate the latter, we have used the LO DAS parton densities presented in Sec. III B. Note that the results 


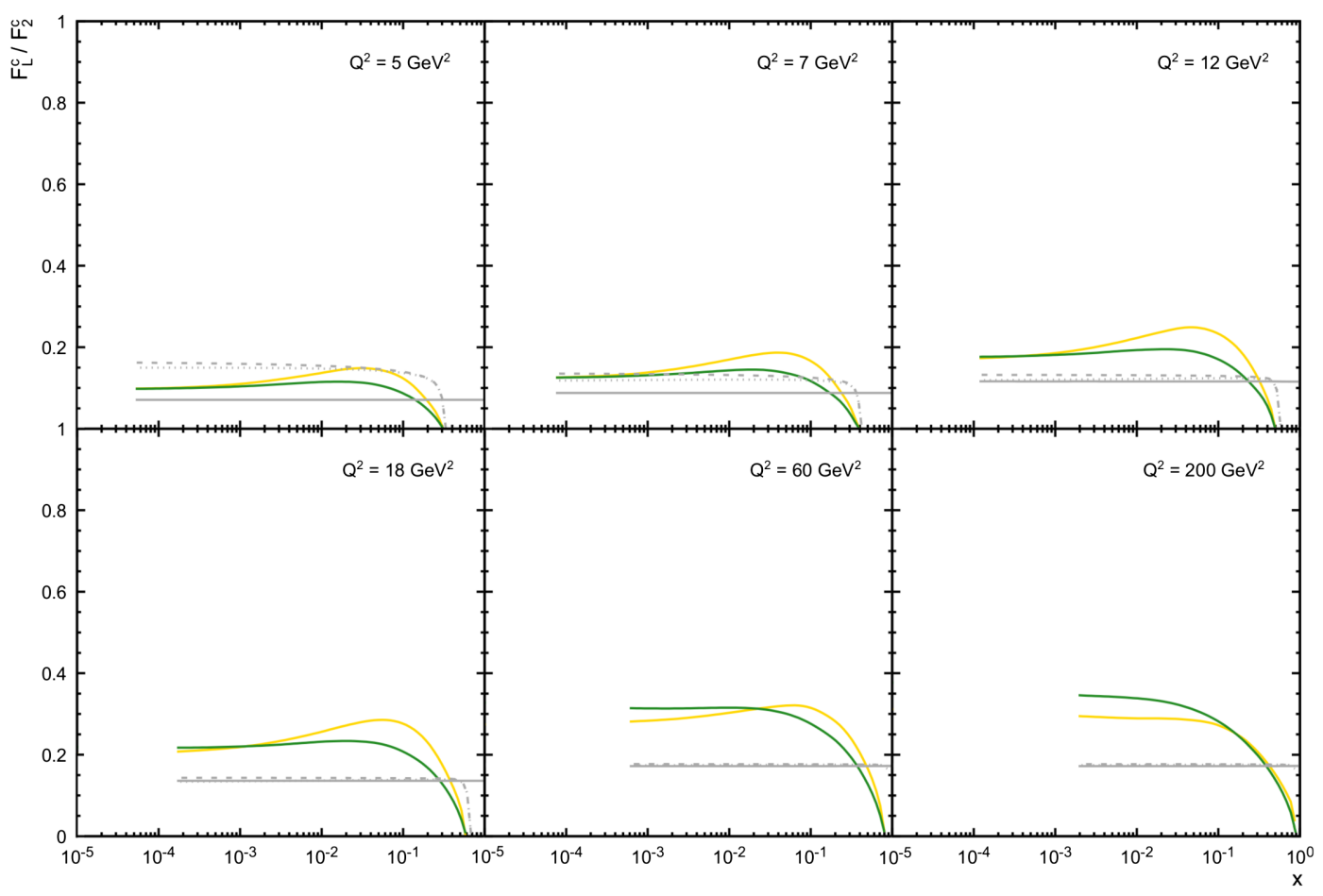

FIG. 5. The ratio $\hat{R}^{c}\left(x, Q^{2}\right)$ as a function of $x$ calculated at different $Q^{2}$ values. Notation of green and yellow solid curves is the same as in Fig. 1. The collinear results for $\hat{R}_{\mathrm{LO}}^{c}\left(x, Q^{2}\right), \hat{R}_{\mathrm{NLO}}^{c}\left(x, Q^{2}\right)$ and $\hat{R}_{\mathrm{NNLO}}^{c}\left(x, Q^{2}\right)$ are represented by solid, dashed and dotted gray curves, respectively.

for this ratio can be found in [73], using the powerlike behavior $x^{-\Delta}$ of the collinear PDFs.

Our calculations show that the $k_{T}$-factorization predictions rather close to the results obtained beyond $\mathrm{LO}$ of the collinear perturbation theory. This is in complete agreement with the usual statement about the property of $k_{T}$-factorization, which resums the main part of higher order $\mathrm{pQCD}$ contributions at the small $x$. Indeed, the LO results obtained in the collinear perturbation theory lead to too small values for the ratio $\hat{R}^{c}\left(x, Q^{2}\right)$. Moreover, the LO collinear Wilson coefficients have no singularities at the first Mellin moment; thus the collinear PDFs cancel out exactly with respect to $\hat{R}^{c}\left(x, Q^{2}\right)$ (see discussions in Appendix B). The corresponding NLO and NNLO collinear Wilson coefficients have singularities at the first Mellin moment and thus lead to the $x$-dependence of the ratio $\hat{R}^{c}\left(x, Q^{2}\right)$. However, the $Q^{2}$-dependence of the ratio $\hat{R}^{c}\left(x, Q^{2}\right)$ in the NLO and NNLO results are noticeably different from the corresponding $Q^{2}$-dependence evaluated with the TMD gluons. In fact, in the $k_{T}$-factorization approach the ratio $\bar{R}^{c}\left(x, Q^{2}\right)$ grows fast when $Q^{2}$ increased whereas in collinear perturbation theory the ratio $\hat{R}^{c}\left(x, Q^{2}\right)$ grows slowly. Moreover, at the large $Q^{2}$ values $(Q 2 \geq$ $12 \mathrm{GeV}^{2}$ ) collinear results become practically independent of the orders of perturbation theory, since the coupling constant becomes very small.

Of course, the difference between the predicted $\bar{R}^{c}\left(x, Q^{2}\right)$ and $\hat{R}^{c}\left(x, Q^{2}\right)$ ratios at moderate and large $Q^{2}$ is unclear especially because there are no experimental data for the $\mathrm{SF} F_{L}^{c}\left(x, Q^{2}\right)$ and, accordingly, for the ratio $\bar{R}^{c}\left(x, Q^{2}\right)$. Indeed, the $k_{T}$-factorization with the estimated $\bar{R}^{c}\left(x, Q^{2}\right)$ leads to a good agreement between experimental data and theoretical predictions for both reduced cross sections $\sigma_{\text {red }}^{c \bar{c}}$ and $\operatorname{SF} F_{2}^{c}\left(x, Q^{2}\right)$, as one can see in Figs. 1 and 3 . From another side, the experimental data for both $\sigma_{\text {red }}^{c \bar{c}}$ and $F_{2}^{c}\left(x, Q^{2}\right)$ are in good agreement with the corresponding theoretical predictions obtained in the framework of collinear approach [1,37-39] (see also Sec. II.5 in the recent review [46]). However, we would like to notice that there is a quite similar situation between the exclusive reduced cross section $\sigma_{\text {red }}\left(x, Q^{2}\right)$ and $F_{2}\left(x, Q^{2}\right)$ : experimental data for both observables are in good agreement with the corresponding theoretical predictions (see [74] and discussions therein), where the calculated $\mathrm{SF} F_{L}\left(x, Q^{2}\right)$ is known to be very sensitive to low $x$ resummation (see, for example, Sec. IX.3 in the recent review [46]). But, apart of $F_{L}^{c}\left(x, Q^{2}\right)$, SF $F_{L}\left(x, Q^{2}\right)$ is measured at the HERA (see [75] and references therein). Therefore, it seems that in order to understand the difference between the predictions for $\bar{R}^{c}\left(x, Q^{2}\right)$ and $\hat{R}^{c}\left(x, Q^{2}\right)$ ratios at large $Q^{2}$ one has to investigate the $\mathrm{SF} F_{L}\left(x, Q^{2}\right)$ using the same approaches, which is out of the scope of our present work. We plan to perform such investigation in forthcoming study and then return to the study of the heavy quark parts and, correspondingly, the ratio $\bar{R}^{c}\left(x, Q^{2}\right)$. 


\section{CONCLUSIONS}

We have studied the heavy quark production processes using the transverse momentum dependent gluon distribution function in a proton obtained recently [29] using the Kimber-Martin-Ryskin prescription from the Besselinspired behavior of parton densities at small Bjorken $x$ values. The Bessel-like behavior of parton densities at small Bjorken $x$ was obtained [30-33] in-turn in the case of the flat initial conditions for DGLAP evolution equations in the double scaling QCD approximation. To construct the TMD parton distributions we implemented [29] the different treatments of the kinematical constraint reflecting the angular and strong ordering conditions, and discussed the relations between the differential and integral formulation of the KMR approach. Additionally, we have tested the TMD gluon density obtained from the numerical solution of the CCFM evolution equation, which smoothly interpolates between the small $x$ BFKL dynamics and large- $x$ DGLAP ones.

We have considered the (reduced) cross sections $\sigma_{\text {red }}^{Q \bar{Q}}$ (where $Q=c, b$ ) and charm and beauty contributions to the deep inelastic proton SFs $F_{2}\left(x, Q^{2}\right)$ and $F_{L}\left(x, Q^{2}\right)$. To show the importance of the longitudinal structure function $F_{L}^{c}\left(x, Q^{2}\right)$ and $F_{L}^{b}\left(x, Q^{2}\right)$, we compare the results for $\sigma_{\text {red }}^{c \bar{c}}$ and $\sigma_{\text {red }}^{b \bar{b}}$ with the SFs and $F_{2}^{c}\left(x, Q^{2}\right)$ and $F_{2}^{b}\left(x, Q^{2}\right)$. We achieved a good agreement between the HERA experimental data for these observables and our theoretical predictions and demonstrated the importance of the contributions of $F_{L}^{c}\left(x, Q^{2}\right)$ and $F_{L}^{b}\left(x, Q^{2}\right)$ at small $x$. Concerning the ratio of the proton SFs, namely, $\bar{R}^{c}\left(x, Q^{2}\right)=$ $F_{L}^{c}\left(x, Q^{2}\right) / F_{2}^{c}\left(x, Q^{2}\right)$, we show that the results of $k_{T^{-}}$ factorization calculations are similar to the ones obtained beyond LO of collinear perturbation theory. This effect is clearly visible for $Q^{2} \leq 12 \mathrm{GeV}^{2}$. However, starting with $Q^{2} \geq 12 \mathrm{GeV}^{2}$, the $k_{T}$-factorization leads to larger values for the ratio $\hat{R}^{c}\left(x, Q^{2}\right)$, which needs additional investigations.

As we discussed already in Sec. IV B, in the next step we plan to study the longitudinal structure function $F_{L}\left(x, Q^{2}\right)$ and compare the results with the previous ones $[61,76]$ and $[59,77,78]$ obtained in the framework of $k_{T}$-factorization and collinear perturbation theory, respectively. This study is important in itself and will provide some clues to solve the problem of differences in the predictions for the ratio $\bar{R}^{c}\left(x, Q^{2}\right)$ obtained between the framework of $k_{T}$-factorization approach and conventional (collinear) QCD factorization (see Sec. IV B).

Moreover, we plan to extend the present analysis beyond the LO approximation, in order to obtain the results for the NLO TMD parton densities using the corresponding NLO results [30-32] for the standard PDFs in the generalized DAS approach. We will check also the results for the NLO matrix elements (see [27,79] and references and discussions therein). These results seem to be extremely important for future experiments, in particular, at the Electron-Ion Collider (EIC) and Electron-Ion Collider in China (EiCC) (see $[80,81]$ and discussions and references therein). With the EIC, an essential low $x$ (up to $x \sim 10^{-4}$ ) region is expected to be probed, thus providing us with new and precise data for DIS SFs, especially data for longitudinal SF $F_{L}\left(x, Q^{2}\right)$. The EiCC could provide a new information on the light and sea quark density in a proton, which is, of course, important to produce and update the theoretical high-order predictions for $F_{L}\left(x, Q^{2}\right)$. Moreover, EIC and EiCC measurements could be important to distinguish between the different noncollinear QCD evolution scenarios widely discussed at present (see, for example, review [2]).

\section{ACKNOWLEDGMENTS}

We thank G. R. Boroun for important comments and remarks. A. V. L. also thanks H. Jung, S. P. Baranov and M. A. Malyshev for very useful discussions. A. V. L. is grateful to DESY Directorate for the support in the framework of Cooperation Agreement between MSU and DESY on the phenomenology of the LHC processes and TMD parton densities. P. M. Z. is supported in part by the National Natural Science Foundation of China (Grants No. 11975320).

\section{APPENDIX A: COLLINEAR APPROACH}

It is easy to obtain the following results in the collinear generalized DAS approach (see [82,83]):

$$
\hat{F}_{k}^{Q}\left(x, Q^{2}\right)=C_{k, g}\left(x, Q^{2}, m_{Q}^{2}\right) \otimes f_{g}\left(x, Q^{2}\right),
$$

where the SFs obtained in the generalized DAS approach, were marked as $\hat{F}_{k}^{Q}\left(x, Q^{2}\right)$. Here $\otimes$ is the Mellin convolution,

$$
\begin{aligned}
C_{k, g} & \left(x, Q^{2}, m_{f}^{2}\right) \otimes f_{g}\left(x, Q^{2}\right) \\
= & \int_{x / x_{2}}^{1} \frac{d y}{y} C_{k, g}\left(x / y, Q^{2}, m_{f}^{2}\right) f_{g}\left(y, Q^{2}\right) \\
& =\int_{x}^{x_{2}} \frac{d y}{y} C_{k, g}\left(y, Q^{2}, m_{f}^{2}\right) f_{g}\left(x / y, Q^{2}\right),
\end{aligned}
$$

where

$$
x_{2}=x_{1}(b=0)=\frac{1}{1+4 a_{Q}} .
$$

It can be represented as

$$
\hat{R}^{Q}\left(x, Q^{2}\right)=\frac{\hat{F}_{L}^{Q}\left(x, Q^{2}\right)}{\hat{F}_{2}^{Q}\left(x, Q^{2}\right)}=\frac{C_{L, g}\left(x, Q^{2}, m_{Q}^{2}\right) \otimes f_{g}\left(x, Q^{2}\right)}{C_{2, g}\left(x, Q^{2}, m_{Q}^{2}\right) \otimes f_{g}\left(x, Q^{2}\right)},
$$


where $f_{g}\left(x, Q^{2}\right)$ is given in (22). In fact, the ratio $\hat{R}^{Q}\left(x, Q^{2}\right)$ depends slowly on nonperturbative input $f_{g}\left(x, Q^{2}\right)$, which contributes to the both numerator and denominator of the ratio $\hat{R}^{Q}\left(x, Q^{2}\right)$.

Using the results of $k_{T}$-factorization and BFKL approach $[5,84]$ (see also [85,86]), the results for the high energy limit of collinear coefficient functions of the heavy quark production process in all orders of perturbation theory were obtained. Thus, using the results [84] below we give formulas for the high energy asymptotic of collinear coefficient functions of the heavy quark production process in the first three orders of the perturbation theory.

\section{LO}

Taking the LO Wilson coefficient (13), results (14) and (15) for on shell coefficient functions and the PDFs considered in the Sec. III B, for the ratio $\hat{R}_{\mathrm{LO}}^{Q}\left(x, Q^{2}\right)$ we have

$$
\hat{R}_{\mathrm{LO}}^{Q}\left(x, Q^{2}\right)=\frac{B_{L, g}^{(0)}\left(x, Q^{2}, m_{Q}^{2}\right) \otimes f_{g}\left(x, Q^{2}\right)}{B_{2, g}^{(0)}\left(x, Q^{2}, m_{Q}^{2}\right) \otimes f_{g}\left(x, Q^{2}\right)},
$$

where the dependence of gluon density $f_{g}\left(x, Q^{2}\right)$ should be rather weak. In fact, there is no $x$-dependence at all (see Fig. 5), which is associated with the property of the Mellin convolution (A2) in the low $x$ region (see Appendix B).

\section{NLO}

Through NLO, we have

$$
C_{k, g}(x)=e_{Q}^{2} a_{s}\left(\mu^{2}\right)\left[B_{k, g}^{(0)}(x, a)+a_{s}\left(\mu^{2}\right) B_{k, g}^{(1)}(x, a)\right] .
$$

The NLO coefficient functions $B_{k, g}^{(1)}(x, a)$ of photon-gluon fusion subprocess are rather lengthy and only available as computer codes [87]. Following [82,83], it is sufficient to work in the high energy regime, defined by $x \ll 1$, where the compact form was assumed ${ }^{3}$ [84-86],

$$
\begin{aligned}
B_{k, g}^{(1)}(x, a) & =\beta\left[R_{k, g}^{(1)}(1, a)+4 C_{A} B_{k, g}^{(0)}(1, a) L_{\mu}\right], \\
L_{\mu} & =\ln \frac{M^{2}}{\mu^{2}}, \quad M^{2}=4 m^{2},
\end{aligned}
$$

with

\footnotetext{
${ }^{3}$ Following Ref. [84], we will use the case $M^{2}=4 m^{2}$ in the collinear approach. We would like to note that in the original papers $[85,86]$ the scale $M^{2}=m^{2}$ has been used, which is inconsistent with the results in Eqs. (A10), (A11) and (A18).
}

$$
\begin{aligned}
R_{2, g}^{(1)}(1, a)= & \frac{8}{9} C_{A}[5+(13-10 a) J(a)+6(1-a) I(a)] \\
R_{L, g}^{(1)}(1, a)= & -\frac{16}{9} C_{A} x_{2}\{1-12 a-[3+4 a(1-6 a)] J(a) \\
& +12 a(1+3 a) I(a)\}
\end{aligned}
$$

and

$$
\begin{aligned}
& B_{2, g}^{(0)}(1, a)=\frac{2}{3}[1+2(1-a) J(a)] \\
& B_{L, g}^{(0)}(1, a)=\frac{4}{3} x_{2}[1+6 a-4 a(1+3 a) J(a)]
\end{aligned}
$$

where $^{4}$

$$
\begin{gathered}
J(a)=-\sqrt{x_{2}} \ln t, \quad t=\frac{1-\sqrt{x_{2}}}{1+\sqrt{x_{2}}}, \quad(\mathrm{~A} \\
I(a)=-\sqrt{x_{2}}\left[\zeta(2)+\frac{1}{2} \ln ^{2} t-\ln \left(a x_{2}\right) \ln t+2 \mathrm{Li}_{2}(-t)\right]
\end{gathered}
$$

with

$$
\mathrm{Li}_{2}(x)=-\int_{0}^{1} \frac{d y}{y} \ln (1-x y)
$$

being the dilogarithmic function. We would like to mention that $B_{k, g}^{(0)}(1, a)$ is the first moment of the LO Wilson coefficients $B_{k, g}^{(0)}(x, a)$ [see (14) and (15)],

$$
B_{k, g}^{(0)}(n, a)=\int_{0}^{x_{2}} d x x^{n-2} B_{k, g}^{(0)}(x, a) .
$$

So, at the NLO for the ratio $\hat{R}^{Q}\left(x, Q^{2}\right)$ we have

$\hat{R}_{\mathrm{NLO}}^{Q}\left(x, Q^{2}\right)=\frac{\left[B_{L, g}^{(0)}(x, a)+a_{s}\left(\mu^{2}\right) B_{L, g}^{(1)}(x, a)\right] \otimes f_{g}\left(x, Q^{2}\right)}{\left[B_{2, g}^{(0)}(x, a)+a_{s}\left(\mu^{2}\right) B_{2, g}^{(1)}(x, a)\right] \otimes f_{g}\left(x, Q^{2}\right)}$,

where the LO gluon density $f_{g}\left(x, Q^{2}\right)$ given by (22) is used because its contribution to the ratio $\hat{R}_{\mathrm{NLO}}^{Q}\left(x, Q^{2}\right)$ is strongly suppressed (see Appendix B).

\section{NNLO}

Following to the results [86], for the coefficient function we have

\footnotetext{
${ }^{4}$ The functions $J(a)$ and $I(a)$ in (A10) and (A11) coincide with ones in [82] and differ from ones in [84-86] by an additional factor $4 a$. The function $K(a)$ in (A18) coincides with the combination $4 a\left[K(a)+\ln \left(4 a x_{2}\right) I(a)\right]$.
} 


$$
C_{k, g}(x)=e_{Q}^{2} a_{s}\left(\mu^{2}\right)\left[B_{k, g}^{(0)}(x, a)+a_{s}\left(\mu^{2}\right) B_{k, g}^{(1)}(x, a)+a_{s}^{2}\left(\mu^{2}\right) B_{k, g}^{(2)}(x, a)\right],
$$

where the coefficient $B_{k, g}^{(2)}(x, a)$ has the compact form in the high energy regime,

$$
B_{k, g}^{(2)}(x, a)=\beta \ln (1 / x)\left[R_{k, g}^{(2)}(1, a)+4 C_{A} R_{k, g}^{(1)}(1, a) L_{\mu}+8 C_{A}^{2} B_{k, g}^{(0)}(1, a) L_{\mu}^{2}\right]+O\left(x^{0}\right),
$$

with

$$
\begin{aligned}
R_{2, g}^{(2)}(1, a)= & \frac{32}{27} C_{A}^{2}[46+(71-92 a) J(a)+3(13-10 a) I(a)-9(1-a) K(a)], \\
R_{L, g}^{(1)}(1, a)= & \frac{64}{27} C_{A}^{2} x_{2}\left\{34+240 a-\left[3+136 a+480 a^{2}\right] J(a)+3[3+4 a(1-6 a)] I(a)\right. \\
& +18 a(1+3 a) K(a)\},
\end{aligned}
$$

where $J(a)$ and $I(a)$ are defined by (A10) and (A11), respectively, and

$$
\begin{aligned}
K(a)= & -\sqrt{x_{2}}\left[4\left(\zeta(3)+\mathrm{Li}_{3}(-t)-\mathrm{Li}_{2}(-t) \ln t-2 S_{1,2}(-t)\right)+2 \ln \left(a x_{2}\right)\right. \\
& \left.\times\left(\zeta(2)+2 \mathrm{Li}_{2}(-t)\right)-\frac{1}{3} \ln ^{3} t-\ln ^{2}\left(a x_{2}\right) \ln t+\ln \left(a x_{2}\right) \ln ^{2} t\right]
\end{aligned}
$$

where $t$ is given in (A10) and

$$
\mathrm{Li}_{3}(x)=\int_{0}^{1} \frac{d y}{y} \ln (y) \ln (1-x y), \quad S_{1,2}(x)=\frac{1}{2} \int_{0}^{1} \frac{d y}{y} \ln ^{2}(1-x y)
$$

are the trilogarithmic function $\mathrm{Li}_{3}(x)$ and Nilsen Polylogarithm $S_{1,2}(x)$ (see [88]). The results for $K(a)$ in the form of harmonic Polylogarithms [89] can be found in [86].

So, at the NNLO for the ratio $\hat{R}^{Q}\left(x, Q^{2}\right)$ we have

$$
\hat{R}_{\mathrm{NNLO}}^{Q}\left(x, Q^{2}\right)=\frac{\left[B_{L, g}^{(0)}(x, a)+a_{s}\left(\mu^{2}\right) B_{L, g}^{(1)}(x, a)+a_{s}^{2}\left(\mu^{2}\right) B_{L, g}^{(2)}(x, a)\right] \otimes f_{g}\left(x, Q^{2}\right)}{\left[B_{2, g}^{(0)}(x, a)+a_{s}\left(\mu^{2}\right) B_{2, g}^{(1)}(x, a)+a_{s}^{2}\left(\mu^{2}\right) B_{2, g}^{(2)}(x, a)\right] \otimes f_{g}\left(x, Q^{2}\right)},
$$

where the LO gluon density $f_{g}\left(x, Q^{2}\right)$ given by (22) is used because its contribution to the ratio $\hat{R}_{\mathrm{NNLO}}^{Q}\left(x, Q^{2}\right)$ is strongly suppressed (see Appendix B).

\section{APPENDIX B: COLLINEAR RESULTS IN DAS APPROACH}

The use of the DAS approach for the PDFs makes it possible to simplify the formulas for the relation $\hat{R}^{Q}\left(x, Q^{2}\right)$ significantly. We will show this below.

Taking the results (14) and (15) for on shell coefficient functions and the PDFs considered in the Sec. III B, it is easily to obtain the following LO results in the generalized DAS approach (see [82]):

$$
\hat{F}_{k}^{Q}\left(x, Q^{2}\right)=M_{k, g}\left(1, Q^{2}, m_{Q}^{2}\right) f_{g}\left(x, Q^{2}\right),
$$

where $M_{k, g}\left(1, Q^{2}, m^{2}\right)$ is the first Mellin moment $(n=1)$ [see (A10)]. The Mellin moments can be defined as

$$
M_{k, g}\left(n, Q^{2}, m_{Q}^{2}\right)=\int_{0}^{x_{2}} d x x^{n-2} C_{k}^{g}\left(x, Q^{2}, m_{Q}^{2}\right)
$$

where $x_{2}$ is given by (A3). In fact, the nonperturbative input $f_{g}\left(x, Q^{2}\right)$ does cancel in the $\hat{R}_{f}$ ratio, and we have 


$$
\hat{R}^{Q}\left(x, Q^{2}\right)=\frac{\hat{F}_{L}^{Q}\left(x, Q^{2}\right)}{\hat{F}_{2}^{Q}\left(x, Q^{2}\right)}=\frac{M_{L, g}\left(1, Q^{2}, m_{Q}^{2}\right)}{M_{2, g}\left(1, Q^{2}, m_{Q}^{2}\right)} .
$$

In this case the moments $M_{k, g}\left(1, Q^{2}, m_{Q}^{2}\right)$ have no singularities at $n \rightarrow 1$.

\section{LO}

Taking the integral (B2) which leads to (A13) at the LO, we can obtain the results (A9), using (see [82]) the following auxiliary formulas ${ }^{5}$ :

$$
\begin{gathered}
\int_{0}^{x_{2}} d x x^{m} \beta= \begin{cases}1-2 a J(a), & \text { if } m=0 \\
\frac{x_{2}}{2}[1+6 a-4 a(1+3 a) J(a)], & \text { if } m=1 \\
\frac{x_{2}^{2}}{3}\left[(1+3 a)(1+10 a)-6 a\left(1+6 a+10 a^{2}\right) J(a)\right], & \text { if } m=2\end{cases} \\
\int_{0}^{b} d x x^{m} L(\beta)= \begin{cases}J(a), & \text { if } m=0 \\
-\frac{x_{2}}{2}[1-(1+2 a) J(a)], & \text { if } m=1 \\
-\frac{x_{2}^{2}}{6}\left[3(1+2 a)-2\left(1+4 a+6 a^{2}\right) J(a)\right], & \text { if } m=2 .\end{cases}
\end{gathered}
$$

So, at the LO the small- $x$ approximation formula (A5) reads

$\hat{R}_{\mathrm{LO}}^{Q}\left(x, Q^{2}\right)=2 x_{2} \frac{1+6 a_{Q}-4 a_{Q}\left(1+3 a_{Q}\right) J\left(a_{Q}\right)}{1+2\left(1-a_{Q}\right) J\left(a_{Q}\right)}$,

which is $x$-independent, in full agreement with the numerical evaluation of the $\bar{R}_{\mathrm{LO}}^{Q}\left(x, Q^{2}\right)$ in (A5).

\section{NLO}

At NLO, the coefficient function $C_{k}^{g}(x)$ has the form (A6) with the NLO coefficients $B_{k, g}^{(1)}(x, a)$ given by (A7) and (A8). Its moments $M_{k, g}\left(n, Q^{2}, \mu^{2}\right)$ exhibit the corresponding structure,

$M_{k, g}\left(n, Q^{2}, \mu^{2}\right)=e_{Q}^{2} a_{s}\left(\mu^{2}\right)\left[B_{k, g}^{(0)}(n, a)+a_{s}\left(\mu^{2}\right) B_{k, g}^{(1)}(n, a)\right]$.

The Mellin transforms of $B_{k, g}^{(1)}(x, a)$ exhibit singularities in the limit $n \rightarrow 1$, which lead to modifications in (B1). As was shown [90], the terms involving $1 / \delta$ at $n=1+\delta \rightarrow 1$ [which correspond to singularities of the Mellin moments $M_{k, g}(n)$ (see (B2)] at $n \rightarrow 1$ ) depend on the exact form of the asymptotic low- $x$ behavior encoded in $f_{g}\left(x, \mu^{2}\right)$. Using

\footnotetext{
${ }^{5}$ In the original paper [82] the second result in (B4) was presented with an error "... $1-2 a \ldots$. instead of the correct expression "... 1+6a ..," and the third result in (B5) was presented with an error " $\ldots x_{2}^{2} / 3 \ldots$ " instead of the correct expression “... $x_{2}^{2} / 6 \ldots$..
}

the results for $f_{g}\left(x, \mu^{2}\right)$ from (22), we obtain the modification (see [82] and discussions therein),

$$
\frac{1}{\delta} \rightarrow \frac{1}{\tilde{\delta}_{ \pm}}, \quad \frac{1}{\tilde{\delta}_{+}(x)} \approx \frac{1}{\rho_{g}(x, \mu)}, \quad \frac{1}{\tilde{\delta}_{-}(x)} \approx \ln \frac{1}{x},
$$

where $\rho_{g}(x, \mu)$ are given by (27).

Because the ratio $f_{g}^{-}\left(x, Q^{2}\right) / f_{g}^{+}\left(x, Q^{2}\right)$ is rather small at the $Q^{2}$ values considered, the expression (B1) is modified to become

$$
\hat{F}_{k}^{Q}\left(x, Q^{2}\right) \approx \tilde{M}_{k, g}\left(1, \mu^{2}, a\right) x f_{g}\left(x, \mu^{2}\right),
$$

where $\tilde{M}_{k, g}\left(1, \mu^{2}\right)$ is obtained from $M_{k, g}\left(n, \mu^{2}\right)$ by taking the limit $n \rightarrow 1$ and replacing $1 /(n-1) \rightarrow 1 / \tilde{\delta}_{+}$in $B_{k, g}^{(1)}(n, a)$. Consequently, one needs to substitute only

$$
B_{k, g}^{(1)}(1, a) \rightarrow \tilde{B}_{k, g}^{(1)}(1, a)
$$

in the NLO part of (B16), i.e.,

$$
\tilde{M}_{k, g}\left(1, Q^{2}, \mu^{2}\right)=e_{Q}^{2} a_{s}\left(\mu^{2}\right)\left[B_{k, g}^{(0)}(1, a)+a_{s}\left(\mu^{2}\right) \tilde{B}_{k, g}^{(1)}(1, a)\right] .
$$

Using the identity,

$$
\begin{aligned}
& \frac{1}{I_{0}(\sigma(\hat{x}))} \int_{\hat{x}}^{1} \frac{d y}{y} \beta\left(\frac{x}{y}\right) I_{0}(\sigma(y)) \\
& \approx \frac{1}{\tilde{\delta}_{+}(\hat{x})}+\phi_{1}(a) \equiv \frac{1}{\hat{\delta}_{+}}, \quad \hat{x}=\frac{x}{x_{2}},
\end{aligned}
$$


where

$$
\phi_{1}(a)=-\ln \left(a x_{2}\right)-\hat{J}(a), \quad \hat{J}(a)=\frac{J(a)}{x_{2}},
$$

we find the Mellin transform (B2) of (A7),

$$
\tilde{B}_{k, g}^{(1)}(1, a) \approx \frac{1}{\hat{\delta}_{+}}\left[R_{k, g}^{(1)}(1, a)+4 C_{a} B_{k, g}^{(0)}(1, a) L_{\mu}\right]
$$

where $R_{k, g}^{(1)}(1, a)$ and $B_{k, g}^{(0)}(1, a)$ are given in (A8) and (A9), respectively.

So, at the NLO for the ratio $\hat{R}^{Q}\left(x, Q^{2}\right)$ we have

$$
\hat{R}_{\mathrm{NLO}}^{Q}\left(x, Q^{2}\right) \approx \frac{\tilde{M}_{L, g}\left(1, Q^{2}, m_{f}^{2}\right)}{\tilde{M}_{2, g}\left(1, Q^{2}, m_{f}^{2}\right)}=\frac{B_{L, g}^{(0)}(1, a)+a_{s}\left(\mu^{2}\right) \tilde{B}_{L, g}^{(1)}(1, a)}{B_{2, g}^{(0)}(1, a)+a_{s}\left(\mu^{2}\right) \tilde{B}_{2, g}^{(1)}(1, a)},
$$

where the ratio has some $x$-dependence coming from the corresponding $x$-dependence of $\hat{\delta}_{+}$in (B12). The $x$-dependence is in rather good agreement with the numerical results in (A20).

\section{NNLO}

At NNLO, the coefficient function $C_{k}^{g}(x)$ has the form (A15) with the NNLO coefficients $B_{k, g}^{(2)}(x, a)$ given in Eqs. (A16) and (A17). Its moment $M_{2, g}\left(n, Q^{2}, \mu^{2}\right)$ exhibits the corresponding structure,

$$
M_{k, g}\left(n, Q^{2}, \mu^{2}\right)=e_{Q}^{2} a_{s}\left(\mu^{2}\right)\left[B_{k, g}^{(0)}(n, a)+a_{s}\left(\mu^{2}\right) B_{k, g}^{(1)}(n, a)+a_{s}^{2}\left(\mu^{2}\right) B_{k, g}^{(2)}(n, a)\right] .
$$

The Mellin transforms of $B_{k, g}^{(2)}(x, a)$ exhibit singularities in the limit $n \rightarrow 1$, which have the form,

$$
\frac{1}{(n-1)^{2}} \rightarrow \frac{1}{\tilde{\delta}_{++}^{2}}=\frac{1}{\rho^{2}(x, \mu)} \frac{I_{2}(\sigma)}{I_{0}(\sigma)}
$$

where all definitions can be found in (7) and (23). So, by analogy with the NLO case, we have

$$
\tilde{M}_{k, g}\left(1, Q^{2}, \mu^{2}\right)=e_{Q}^{2} a_{s}\left(\mu^{2}\right)\left[B_{k, g}^{(0)}(1, a)+a_{s}\left(\mu^{2}\right) \tilde{B}_{k, g}^{(1)}(1, a)+a_{s}^{2}\left(\mu^{2}\right) \tilde{B}_{k, g}^{(2)}(1, a)\right] .
$$

Using the identity,

$$
\frac{1}{I_{0}(\sigma(\hat{x}))} \int_{\hat{x}}^{1} \frac{d y}{y} \beta\left(\frac{x}{y}\right) \ln \left(\frac{y}{x}\right) I_{0}(\sigma(y)) \approx \frac{1}{\tilde{\delta}_{++}^{2}(\hat{x})} \equiv \frac{1}{\hat{\delta}_{++}^{2}}, \quad \hat{x}=\frac{x}{x_{1}},
$$

we find the Mellin transform (B2) of (A7),

$$
\tilde{B}_{k, g}^{(2)}(1, a) \approx \frac{1}{\hat{\delta}_{++}^{2}}\left[R_{k, g}^{(2)}(1, a)+4 C_{A} R_{k, g}^{(1)}(1, a) L_{\mu}+8 C_{A}^{2} B_{k, g}^{(0)}(1, a) L_{\mu}^{2}\right]
$$

with $R_{k, g}^{(2)}(1, a), R_{k, g}^{(1)}(1, a)$ and $B_{k, g}^{(0)}(1, a)$ are given in (A17), (A8) and (A9), respectively.

So, for the ratio $\hat{R}^{Q}\left(x, Q^{2}\right)$ we have

$$
\hat{R}_{\mathrm{NNLO}}^{Q}\left(x, Q^{2}\right) \approx \frac{B_{L, g}^{(0)}(1, a)+a_{s}\left(\mu^{2}\right) \tilde{B}_{L, g}^{(1)}(1, a)+a_{s}^{2}\left(\mu^{2}\right) \tilde{B}_{L, g}^{(2)}(1, a)}{B_{2, g}^{(0)}(1, a)+a_{s}\left(\mu^{2}\right) \tilde{B}_{2, g}^{(1)}(1, a)+a_{s}^{2}\left(\mu^{2}\right) \tilde{B}_{2, g}^{(2)}(1, a)},
$$

where the ratio has some $x$-dependence coming from the corresponding $x$-dependence of $\hat{\delta}_{++}$in (B19). The $x$-dependence is in rather good agreement with the numerical results in (A20). 
[1] H. Abramowicz et al. (H1 and ZEUS Collaborations), Eur. Phys. J. C 78, 473 (2018).

[2] R. Angeles-Martinez et al., Acta Phys. Pol. B 46, 2501 (2015).

[3] N. A. Abdulov et al., Eur. Phys. J. C 81, 752 (2021).

[4] J. C. Collins, D. E. Soper, and G. F. Sterman, Nucl. Phys. B223, 381 (1983); 250, 199 (1985).

[5] S. Catani, M. Ciafaloni, and F. Hautmann, Nucl. Phys. B366, 135 (1991).

[6] J. C. Collins and R. K. Ellis, Nucl. Phys. B360, 3 (1991).

[7] L. V. Gribov, E. M. Levin, and M. G. Ryskin, Phys. Rep. 100, 1 (1983); E. M. Levin, M. G. Ryskin, Yu. M. Shabelsky, and A. G. Shuvaev, Sov. J. Nucl. Phys. 53, 657 (1991).

[8] E. A. Kuraev, L. N. Lipatov, and V. S. Fadin, Sov. Phys. JETP 44, 443 (1976); 45, 199 (1977); I. I. Balitsky and L. N. Lipatov, Sov. J. Nucl. Phys. 28, 822 (1978).

[9] M. Ciafaloni, Nucl. Phys. B296, 49 (1988); S. Catani, F. Fiorani, and G. Marchesini, Phys. Lett. B 234, 339 (1990); Nucl. Phys. B336, 18 (1990); G. Marchesini, Nucl. Phys. B445, 49 (1995).

[10] A. V. Lipatov, M. A. Malyshev, and H. Jung, Phys. Rev. D 100, 034028 (2019).

[11] S. P. Baranov and A. V. Lipatov, Phys. Rev. D 100, 114021 (2019).

[12] S. P. Baranov and A. V. Lipatov, Phys. Lett. B 785, 338 (2018).

[13] S. P. Baranov, H. Jung, A. V. Lipatov, and M. A. Malyshev, Eur. Phys. J. C 77, 772 (2017).

[14] N. A. Abdulov, A. V. Lipatov, and M. A. Malyshev, Phys. Rev. D 97, 054017 (2018).

[15] A. V. Lipatov, M. A. Malyshev, and N. P. Zotov, Phys. Lett. B 735, 79 (2014).

[16] R. Islam, M. Kumar, and V. S. Rawoot, Eur. Phys. J. C 79, 181 (2019).

[17] A. Szczurek, M. Luszczak, and R. Maciula, Phys. Rev. D 90, 094023 (2014).

[18] H. Jung, M. Krämer, A. V. Lipatov, and N. P. Zotov, Phys. Rev. D 85, 034035 (2012).

[19] H. Jung, M. Krämer, A. V. Lipatov, and N. P. Zotov, J. High Energy Phys. 01 (2011) 085.

[20] S. Dooling, F. Hautmann, and H. Jung, Phys. Lett. B 736, 293 (2014).

[21] F. Hautmann, H. Jung, A. Lelek, V. Radescu, and R. Zlebcik, Phys. Lett. B 772, 446 (2017); J. High Energy Phys. 01 (2018) 070.

[22] M. A. Kimber, A. D. Martin, and M. G. Ryskin, Phys. Rev. D 63, 114027 (2001); G. Watt, A. D. Martin, and M. G. Ryskin, Eur. Phys. J. C 31, 73 (2003).

[23] V. N. Gribov and L. N. Lipatov, Sov. J. Nucl. Phys. 15, 438 (1972); L. N. Lipatov, Sov. J. Nucl. Phys. 20, 94 (1975); G. Altarelli and G. Parisi, Nucl. Phys. B126, 298 (1977); Yu. L. Dokshitzer, Sov. Phys. JETP 46, 641 (1977).

[24] A. D. Martin, M. G. Ryskin, and G. Watt, Eur. Phys. J. C 66, 163 (2010).

[25] R. Abdul Khalek et al. (NNPDF Collaboration), Eur. Phys. J. C 79, 838 (2019).

[26] T. J. Hou et al., arXiv:1908.11394; Phys. Rev. D 103, 014013 (2021).

[27] R. Maciula and A. Szczurek, Phys. Rev. D 100, 054001 (2019).
[28] A. V. Lipatov, M. A. Malyshev, and H. Jung, Phys. Rev. D 101, 034022 (2020).

[29] A. V. Kotikov, A. V. Lipatov, B. G. Shaikhatdenov, and P. Zhang, J. High Energy Phys. 02 (2020) 028.

[30] G. Cvetic, A. Yu. Illarionov, B. A. Kniehl, and A. V. Kotikov, Phys. Lett. B 679, 350 (2009).

[31] A. V. Kotikov and G. Parente, Nucl. Phys. B549, 242 (1999).

[32] A. Yu. Illarionov, A. V. Kotikov, and G. Parente, Phys. Part. Nucl. 39, 307 (2008).

[33] L. Mankiewicz, A. Saalfeld, and T. Weigl, Phys. Lett. B 393, 175 (1997).

[34] A. De Rújula, S. L. Glashow, H. D. Politzer, S. B. Treiman, F. Wilczek, and A. Zee, Phys. Rev. D 10, 1649 (1974).

[35] A. V. Lipatov, M. A. Malyshev, and S. P. Baranov, Eur. Phys. J. C 80, 330 (2020).

[36] K. Golec-Biernat and A. M. Stasto, Phys. Lett. B 781, 633 (2018).

[37] ZEUS Collaboration, J. High Energy Phys. 09 (2014) 127.

[38] H1 Collaboration, Eur. Phys. J. C 71, 1769 (2011); 72, 2252 (2012).

[39] H1 Collaboration, Eur. Phys. J. C 65, 89 (2010).

[40] C. Adloff et al. (H1 Collaboration), Z. Phys. C 72 (1996) 593; Nucl. Phys. B545, 21 (1999).

[41] J. Breitweg et al. (ZEUS Collaboration), Phys. Lett. B 407, 402 (1997); Eur. Phys. J. C 12, 35 (2000).

[42] J. J. Aubert et al. (European Muon Collaboration), Nucl. Phys. B213, 31 (1983); Phys. Lett. 94B, 96 (1980); Phys. Lett. 110B, 73 (1982).

[43] R. Aaij et al. (LHCb Collaboration), Nucl. Phys. B871, 1 (2013); J. High Energy Phys. 06 (2017) 147; J. High Energy Phys. 03 (2016) 159; 09 (2016) 013(E); 05 (2017) 074(E).

[44] A. M. Cooper-Sarkar, R. C. E. Devenish, and A. De Roeck, Int. J. Mod. Phys. A 13, 3385 (1998).

[45] O. Zenaiev, Eur. Phys. J. C 77, 151 (2017).

[46] J. Gao, L. Harland-Lang, and J. Rojo, Phys. Rep. 742, 1 (2018).

[47] M. Cacciari, M. L. Mangano, and P. Nason, Eur. Phys. J. C 75, 610 (2015); O. Zenaiev et al. (PROSA Collaboration), Eur. Phys. J. C 75 (2015), 396; R. Gauld and J. Rojo, Phys. Rev. Lett. 118, 072001 (2017).

[48] A. Cooper-Sarkar, P. Mertsch, and S. Sarkar, J. High Energy Phys. 08 (2011) 042; R. Fiore, L. L. Jenkovszky, A. Kotikov, F. Paccanoni, A. Papa, and E. Predazzi, Phys. Rev. D 68, 093010 (2003); 71, 033002 (2005); 73, 053012 (2006); A. Y. Illarionov, B. A. Kniehl, and A. V. Kotikov, Phys. Rev. Lett. 106, 231802 (2011).

[49] R. Gauld, J. Rojo, L. Rottoli, S. Sarkar, and J. Talbert, J. High Energy Phys. 02 (2016) 130; M. V. Garzelli, S. Moch, O. Zenaiev, A. Cooper-Sarkar, A. Geiser, K. Lipka, R. Placakyte, and G. Sigl (PROSA Collaboration), J. High Energy Phys. 05 (2017) 004.

[50] J. L. Abelleira Fernandez et al. (LHeC Study Group Collaboration), J. Phys. G 39, 075001 (2012); arXiv: 1211.5102; N. Armesto (LHeC Study Group Collaboration), J. Phys. Conf. Ser. 422, 012030 (2013).

[51] M. L. Mangano et al., CERN Yellow Rep. 3, 1 (2017).

[52] A. V. Kotikov, A. V. Lipatov, G. Parente, and N. P. Zotov, Eur. Phys. J. C 26, 51 (2002). 
[53] E. Witten, Nucl. Phys. B104, 445 (1976); M. Gluck and E. Reya, Phys. Lett. 83B, 98 (1979); F. M. Steffens, W. Melnitchouk, and A. W. Thomas, Eur. Phys. J. C 11, 673 (1999).

[54] A. J. Buras, Rev. Mod. Phys. 52, 199 (1980).

[55] A. V. Kotikov and B. G. Shaikhatdenov, Phys. Part. Nucl. 48, 829 (2017); Phys. At. Nucl. 78, 525 (2015); Phys. Part. Nucl. 44, 543 (2013).

[56] B. Badelek, J. Kwiecinski, and A. Stasto, Z. Phys. C 74, 297 (1997).

[57] D. V. Shirkov and I. L. Solovtsov, Phys. Rev. Lett. 79, 1209 (1997); I. L. Solovtsov and D. V. Shirkov, Theor. Math. Phys. 120, 1220 (1999).

[58] G. Cvetic and C. Valenzuela, Braz. J. Phys. 38, 371 (2008); A. P. Bakulev, Phys. Part. Nucl. 40, 715 (2009); N. G. Stefanis, Phys. Part. Nucl. 44, 494 (2013).

[59] A. V. Kotikov, Phys. Lett. B 338, 349 (1994).

[60] Yu. L. Dokshitzer and D. V. Shirkov, Z. Phys. C 67, 449 (1995); S. J. Brodsky, V. S. Fadin, V. T. Kim, L. N. Lipatov, and G. B. Pivovarov, JETP Lett. 70, 155 (1999); Small-x Collaboration, Eur. Phys. J. C 25, 77 (2002).

[61] A. V. Kotikov, A. V. Lipatov, and N. P. Zotov, J. Exp. Theor. Phys. 101, 811 (2005).

[62] A. V. Kotikov, B. G. Shaikhatdenov, and P. Zhang, Phys. Part. Nucl. Lett. 16, 311 (2019).

[63] A. V. Kotikov, B. G. Shaikhatdenov, and P. Zhang, Phys. Rev. D 96, 114002 (2017).

[64] V. A. Matveev, R. M. Muradian, and A. N. Tavkhelidze, Lett. Nuovo Cimento 7, 719 (1973); S. J. Brodsky and G. R. Farrar, Phys. Rev. Lett. 31, 1153 (1973); S. J. Brodsky, J. R. Ellis, E. Gardi, M. Karliner, and M. A. Samuel, Phys. Rev. D 56, 6980 (1997).

[65] C. Lopez and F. J. Yndurain, Nucl. Phys. B171, 231 (1980); B183, 157 (1981); A. Yu. Illarionov, A. V. Kotikov, S. S. Parzycki, and D. V. Peshekhonov, Phys. Rev. D 83, 034014 (2011).

[66] P. Jimenez-Delgado and E. Reya, Phys. Rev. D 89, 074049 (2014); S. Alekhin, J. Blümlein, S. Moch, and R. Placakyte, Phys. Rev. D 96, 014011 (2017).

[67] V. G. Krivokhizhin and A. V. Kotikov, Phys. At. Nucl. 68, 1873 (2005); Phys. Part. Nucl. 40, 1059 (2009); B. G. Shaikhatdenov, A. V. Kotikov, V. G. Krivokhizhin, and G. Parente, Phys. Rev. D 81, 034008 (2010); A. V. Kotikov, V. G. Krivokhizhin, and B. G. Shaikhatdenov, Phys. At. Nucl. 75, 507 (2012); J. Phys. G 42, 095004 (2015); Phys. At. Nucl. 81, 244 (2018).

[68] CMS Collaboration, J. High Energy Phys. 04 (2012) 084.

[69] ATLAS Collaboration, Eur. Phys. J. C 71, 1846 (2011).

[70] F. Hautmann and H. Jung, Nucl. Phys. B883, 1 (2014).

[71] PDG Collaboration, Phys. Rev. D 98, 030001 (2018).

[72] J. Blumlein, On the $k_{T}$-dependent gluon density in hadrons and in the photon, in ?5 QCD and High-Energy Hadronic Interactions. Proceedings, 30th Rencontres de Moriond,
MoriondParticle Physics Meetings, Hadronic Session, Le Arcs, France, March 19-25, 1995 (1995), pp. 191-97. Also in preprint hep-ph/9506446; M. G. Ryskin and Y. M. Shabelski, Z. Phys. C 61, 517 (1994); 66, 151 (1995).

[73] N. Y. Ivanov and B. A. Kniehl, Eur. Phys. J. C 59, 647 (2009); G. R. Boroun, Chin. Phys. C 45, 063105 (2021).

[74] H. Abramowicz et al. (H1 and ZEUS Collaborations), Eur. Phys. J. C 75, 580 (2015); F. D. Aaron et al. (H1 and ZEUS Collaborations), J. High Energy Phys. 01 (2010) 109.

[75] V. Andreev et al. (H1 Collaboration), Eur. Phys. J. C 74, 2814 (2014).

[76] A. V. Kotikov, A. V. Lipatov, and N. P. Zotov, Eur. Phys. J. C 27, 219 (2003).

[77] A. V. Kotikov, J. Exp. Theor. Phys. 80, 979 (1995); A. V. Kotikov and G. Parente, Mod. Phys. Lett. A 12, 963 (1997); J. Exp. Theor. Phys. 85, 17 (1997).

[78] L. P. Kaptari, A. V. Kotikov, N. Y. Chernikova, and P. Zhang, Phys. Rev. D 99, 096019 (2019); JETP Lett. 109, 281 (2019).

[79] A. van Hameren, arXiv:1902.01791; M. Nefedov and V. Saleev, Mod. Phys. Lett. A 32, 1750207 (2017); F. Caporale, F. G. Celiberto, G. Chachamis, D. Gordo Gomez, and A. Sabio Vera, AIP Conf. Proc. 1819, 060009 (2017).

[80] A. Accardi et al., Eur. Phys. J. A 52, 268 (2016).

[81] D. P. Anderle et al., Front. Phys. (Beijing) 16, 64701 (2021).

[82] A. Yu. Illarionov, B. A. Kniehl, and A. V. Kotikov, Phys. Lett. B 663, 66 (2008); Heavy-quark contributions to the ratio $\mathrm{F}(\mathrm{L}) / \mathrm{F}(2)$ at low values of the Bjorken variable $\mathrm{x}$, Proceedings, Helmholz International Summer School on Heavy Quark Physics (2008), pp. 243-252. Also in preprint arXiv:0812.4943.

[83] A. Yu. Illarionov and A. V. Kotikov, Phys. At. Nucl. 75, 1234 (2012).

[84] S. Catani, Z. Phys. C 75, 665 (1997).

[85] S. Catani, M. Ciafaloni, and F. Hautmann, Report No. CERN-Th.6398/92, CERN publishing department, CERN, Geneve; in Proceeding of the Workshop on Physics at HERA, Hamburg (1991), Vol. 2., p. 690, DESY publishing department, DESY, Hamburg; S. Catani and F. Hautmann, Nucl. Phys. B427, 475 (1994); S. Riemersma, J. Smith, and W. L. van Neerven, Phys. Lett. B 347, 143 (1995).

[86] H. Kawamura, N. A. Lo Presti, S. Moch, and A. Vogt, Nucl. Phys. B864, 399 (2012).

[87] E. Laenen, S. Riemersma, J. Smith, and W. L. van Neerven, Nucl. Phys. B392, 162 (1993).

[88] A. Devoto and D. W. Duke, Riv. Nuovo Cimento 7N6, 1 (1984).

[89] E. Remiddi and J. A. M. Vermaseren, Int. J. Mod. Phys. A 15, 725 (2000).

[90] A. V. Kotikov, Phys. Part. Nucl. 38, 1 (2007) 1; 38, 828(E) (2007). 